\title{
Encoder Lipschitz Integers: The Lipschitz integers that have the "division with small remainder" property
}

Ramazan Duram ( $\sim$ rduran@aku.edu.tr)

Department of Mathematics, Afyon Kocatepe University https://orcid.org/0000-0002-8076-0557 Murat Güzeltepe

Department of Mathematics, Sakarya University

\section{Research Article}

Keywords: Quaternion integer, Lipschitz integer, residue class, signal constellation, coding gain

Posted Date: December 7th, 2021

DOI: https://doi.org/10.21203/rs.3.rs-1053517/v2

License: (c) (i) This work is licensed under a Creative Commons Attribution 4.0 International License.

Read Full License 


\title{
Encoder Lipschitz Integers: The Lipschitz in- tegers that have the "division with small re- mainder" property
}

\author{
Ramazan Duran and Murat Guzeltepe
}

\begin{abstract}
The residue class set of a Lipschitz integer is constructed by modulo function with primitive Lipschitz integer whose norm is a prime integer, i.e. prime Lipschitz integer. In this study, we consider primitive Lipschitz integer whose norm is both a prime integer and not a prime integer. If the norm of each element of the residue class set of a Lipschitz integer is less than the norm of the primitive Lipschitz integer used to construct the residue class set of the Lipschitz integer, then, the Euclid division algorithm works for this primitive Lipschitz integer. The Euclid division algorithm always works for prime Lipschitz integers. In other words, the prime Lipschitz integers have the "division with small remainder" property. However, this property is ignored in some studies that have a constructed Lipschitz residue class set that lies on primitive Lipschitz integers whose norm is not a prime integer. In this study, we solve this problem by defining Lipschitz integers that have the "division with small remainder" property, namely, encoder Lipschitz integers set. Therefore, we can define appropriate metrics for codes over Lipschitz integers. Also, we investigate the performances of Lipschitz signal constellations (the left residue class set) obtained by modulo function with Lipschitz integers, which have the "division with small remainder" property, over the additive white Gaussian noise (AWGN) channel by agency of the constellation figure of merit (CFM), average energy, and signal-to-noise ratio (SNR).
\end{abstract}

Keywords. Quaternion integer, Lipschitz integer, residue class, signal constellation, coding gain.

\section{Introduction}

In recent years, many researchers in coding theory have investigated some special code constructions over groups, fields or rings i.e. finite algebraic construction. Specially, they have studied code constructions over finite rings 
of integers and finite fields [1-8]. A Gaussian integer is a complex number that real and imaginary parts are in $\mathbb{Z}$. The set of Gaussian integers that denoted by $\mathbb{Z}[i]$ is shown by $\mathbb{Z}[i]=\left\{\alpha=\alpha_{1}+\alpha_{2} i: \alpha_{1}, \alpha_{2} \in \mathbb{Z}, i^{2}=-1\right\}$. Gaussian integers are a commutative ring and a subset of the complex numbers field, since they are closed under addition and multiplication. Let $\alpha=\alpha_{1}+\alpha_{2} i$ be a Gaussian integer. The conjugate of $\alpha$ is equal to $\bar{\alpha}=\alpha_{1}-\alpha_{2} i$, the norm of $\alpha$ is equal to $N(\alpha)=\alpha_{1}^{2}+\alpha_{2}^{2}$, and the inverse of $\alpha$ is equal to $\alpha^{-1}=\frac{\bar{\alpha}}{N(\alpha)}$ where its norm is non-zero. A Gaussian integer is a prime Gaussian integer if its norm is a prime in $\mathbb{Z}$. A Gaussian integer is a primitive Gaussian integer just if greatest common divisor (gcd) of all components is one i.e. $\operatorname{gcd}\left(\alpha_{1}, \alpha_{2}\right)=1$. Hence, $\alpha_{1}$ and $\alpha_{2}$ are positive integers if $\alpha=\alpha_{1}+\alpha_{2} i$ is a primitive Gaussian integer. In [9], first study about code constructions over Gaussian integers is presented by Huber. In other words, Huber discovered a new way to construct codes for two dimensional signals by virtue of Gaussian integers, i.e. the integral points on the complex plane. The residual class set of the selected Gaussian integer is called a signal constellation. The "signal constellation" is a communication term. In [9], Huber is used prime Gaussian integers such that $1 \equiv p \bmod 4$ where $p=\alpha \bar{\alpha}$, and $\alpha_{1}>\alpha_{2}>0$. Codes over rings of Gaussian integers were studied in papers to [9-12].

The quaternions are a four dimensional vector space that is an algebra over the set of the real numbers $(\mathbb{R})$, and a number system that extends the complex numbers $(\mathbb{C})$. The quaternions are a division algebra that is associative and non-commutative since the multiplication of quaternions has not commutative property. So, $\alpha \beta \neq \beta \alpha$ where $\alpha$ and $\beta$ are quaternions. Let $\alpha=\alpha_{1}+\alpha_{2} i+\alpha_{3} j+\alpha_{4} k$ be a quaternion where $\alpha_{1}$ is real part and $\alpha_{2} i+\alpha_{3} j+\alpha_{4} k$ is imaginary part. Multiplication of quaternions has commutative properties when $\alpha \alpha^{-1}=\alpha^{-1} \alpha=1$, and their imaginary parts are parallel to each other. In [13], Ozen and Guzeltepe study codes over some finite fields by using commutative quaternion integers. The Lipschitz integers are a four-dimensional vector space that is an algebra over the set of integers. The set of Lipschitz integers is forms a subring of quaternion integers ring. A Lipschitz integer is called a primitive Lipschitz integer if the greatest common divisor (gcd) of all components is one i.e. $\operatorname{gcd}\left(\alpha_{1}, \alpha_{2}, \alpha_{3}, \alpha_{4}\right)=1$. In [16], codes over quaternion integers, where the elements are from the commutative ring of even integers, are given. Codes over quaternion integers, and codes over Lipschitz integers were studied in papers given in [13-20]. Without loss of generality in [15],[16], we consider Lipschitz integers, which their norm is both a prime integer and not a prime integer and each component is nonzero, and such that $\alpha_{1} \geq \alpha_{2} \geq \alpha_{3} \geq \alpha_{4}>0$ where $\operatorname{gcd}\left(\alpha_{1}, \alpha_{2}, \alpha_{3}, \alpha_{4}\right)=1$ in this study. In [9], Hubers' original idea is to regard a finite field as a residue class of the Gaussian integer ring modulo a prime Gaussian integer and, by Euclidean division algorithm, to get a unique element of minimal norm in each residue class, which represents each element of a finite field. Therefore, each element of a finite field can be represented by a Gaussian integer with the minimal norm in the residue class. Considering the original idea for a 
residual class of Lipschitz integer rings, any element of the signal constellation may not be represented by a Lipschitz integer with the minimal norm in the residue class. In other words, the norm of any element of the Lipschitz signal constellation is equal to the norm of the primitive Lipschitz integer used to obtained the Lipschitz signal constellation. In this study, we define a set that is consists of primitive Lipschitz integers that have the "division with small remainder" property to solve this problem. This set is named encoder Lipschitz integers set. We construct Lipschitz signal constellations by modulo function with encoder Lipschitz integers. Also, we investigate performances between of Lipschitz integer constellations over the additive white Gaussian noise (AWGN) channel with respect to constellation figure of merit, average energy, and signal-to-noise ratio (SNR). We close this study with the conclusion section.

\section{Preliminaries}

In this section, we give some fundamental information used throughout this paper.

Definition 2.1. The Hamilton quaternion algebra over $\mathbb{R}$, is the associative unital algebra given by the following representation:

i. $\mathbb{H}(\mathbb{R})$ is the free $\mathbb{R}$ - module over the symbols $1, i, j, k$, that is: $\mathbb{H}(\mathbb{R})=$ $\left\{a_{0}+a_{1} i+a_{2} j+a_{3} k: a_{0}, a_{1}, a_{2}, a_{3} \in \mathbb{R}\right\}$,

ii. 1 is the multiplicative identity,

iii. $i^{2}=j^{2}=k^{2}=i j k=-1$,

iv. $i j=-j i=k, j k=-k j=i, k i=-i k=j$.

The definition is natural, in the sense that any unital ring homomorphism $\mathbb{R}_{1} \rightarrow \mathbb{R}_{2}$ extends to a unital ring homomorphism $\mathbb{H}\left(\mathbb{R}_{1}\right) \rightarrow \mathbb{H}\left(\mathbb{R}_{2}\right)$ by mapping 1 to $1, i$ to $i, j$ to $j$ and $k$ to $k$ [21, 2.4.1 Definition]. Let $\alpha=$ $\alpha_{1}+\alpha_{2} i+\alpha_{3} j+\alpha_{4} k$ be a quaternion. Here $\alpha_{1}$ is a real part , and $\alpha_{2} i+\alpha_{3} j+\alpha_{4} k$ is an imaginary part. Also, $\alpha_{1}, \alpha_{2}, \alpha_{2}$, and $\alpha_{2}$ are components of $\alpha$ quaternion. Multiplication of quaternions is non-commutative. But, if the imaginary parts of quaternions are parallel to each other, then multiplication of quaternions is commutative [13]. Also, multiplication of $\alpha$ and $\alpha^{-1}$ is commutative since $\alpha \alpha^{-1}=\alpha^{-1} \alpha=1$.

Definition 2.2. $\alpha=\alpha_{1}+\alpha_{2} i+\alpha_{3} j+\alpha_{4} k$ is a Lipschitz integer just if $\alpha_{1}, \alpha_{2}, \alpha_{3}, \alpha_{4} \in \mathbb{Z}$. The set of all Lipschitz integers that denoted by $\mathcal{H}(\mathbb{Z})$ is written by

$$
\mathcal{H}(\mathbb{Z})=\left\{\alpha_{1}+\alpha_{2} i+\alpha_{3} j+\alpha_{4} k: \alpha_{1}, \alpha_{2}, \alpha_{3}, \alpha_{4} \in \mathbb{Z}\right\} .
$$

This set is a subset of $\mathbb{H}(\mathbb{R}) . \mathcal{H}(\mathbb{Z})$ is forms a subring of the ring of all quaternions because of closed under multiplication and addition. The conjugate of $\alpha$ is $\bar{\alpha}=\alpha_{0}-\alpha_{1} i-\alpha_{2} j-\alpha_{3} k$. The norm of $\alpha$ is $N(\alpha)=\alpha \cdot \bar{\alpha}=\alpha_{0}^{2}+\alpha_{1}^{2}+\alpha_{2}^{2}+\alpha_{3}^{2}$. 
The inverse of $\alpha$ is $\alpha^{-1}=\frac{\bar{\alpha}}{N(\alpha)}$ where $\alpha \neq 0$. The units of $\mathcal{H}(\mathbb{Z})$ under multiplication are $\{ \pm 1, \pm i, \pm j, \pm k\}$. This set has 8 elements. We will use $\mathcal{L}$ notation instead of $\mathcal{H}(\mathbb{Z})$ for the rest of this study.

Definition 2.3. A Lipschitz integer is called a prime Lipschitz integer just if its norm is a prime in $\mathbb{Z}$.

Example 2.1. $\alpha=3-2 i+3 j-k$ is a prime Lipschitz integer since $N(\alpha)=$ $3^{2}+(-2)^{2}+3^{2}+(-1)^{2}=23$ is a prime in $\mathbb{Z}$.

Definition 2.4. $\alpha=\alpha_{1}+\alpha_{2} i+\alpha_{3} j+\alpha_{4} k$ is a primitive Lipschitz integer if greater common divisor (gcd) of each component is equal to 1 . So $\operatorname{gcd}\left(\alpha_{1}, \alpha_{2}, \alpha_{3} j, \alpha_{4}\right)=1$.

Example 2.2. $\alpha=3+4 i+2 j+k$ is a primitive Lipschitz integers because of $\operatorname{gcd}(3,4,2,1)=1$. And so on. Note that, with respect to definition 2.4, components of a Lipschitz integer are positive integers.

Definition 2.5. The nearest integer rounding notation denoted by $\lfloor\cdot 7$ is defined as rounding a rational number to the integer closest to its. For quaternions, each component of a quaternion is separately rounding to the integer closest to its. So, we obtain Lipschitz integers from a quaternions. In this study, the rounding is done in the direction $+\infty$.

Example 2.3. Let be $\alpha=\frac{5}{4}+\frac{1}{2} i-\frac{1}{2} j-\frac{5}{2} k \in \mathbb{H}(\mathbb{R})$ a quaternion. If we use nearest integer rounding notation for $\alpha$, then we obtain a Lipschitz integer. That is,

$$
\begin{aligned}
\lfloor\alpha\rceil & =\left\lfloor\frac{5}{4}+\frac{1}{2} i-\frac{1}{2} j-\frac{5}{2} k\right\rceil \\
& =\left\lfloor\frac{5}{4}\right\rceil+\left\lfloor\frac{1}{2} i\right\rceil+\left\lfloor-\frac{1}{2} j\right\rceil+\left\lfloor-\frac{5}{2} k\right\rceil \\
& =1+1 \cdot i+0 \cdot j+(-2) \cdot k \\
& =1+i-2 k .
\end{aligned}
$$

The residue class set for codes over Gaussian integers that are twodimensional signal space are constructed by the modulo function technique. Similar to Gaussian integers [9], Freudenberger and Shavgulidze generalized this technique for Lipschitz integers whose norm is both a prime integer and not a prime integer [14-15]. This technique, known as the modulo function, is given by the following definition. Note that we consider the primitive Lipschitz integers whose norm is both a prime integer and not a prime integer, and left residue class set of primitive Lipschitz integer in this study.

Definition 2.6. The modulo function $\mu: \mathbb{Z}_{N(\pi)} \rightarrow \mathcal{L}_{\pi}$ is defined by

$$
\mu_{\pi}(z)=z \bmod \pi=z-\pi \cdot\left\lfloor\alpha^{-1} z\right\rceil=z-\pi\left\lfloor\frac{\bar{\pi} z}{N(\pi)}\right\rceil
$$

where $\pi$ is a primitive Lipschitz integer and $z \in \mathbb{Z}_{N(\pi)}$. Here $\mathbb{Z}_{N(\pi)}$ is the well-known residual class ring of ordinary integers with $N(\pi)$ elements, $\mathcal{L}_{\pi}$ is the left residual class set of $z \in \mathbb{Z}_{N(\pi)}$ modulo $\pi$, and $\mu_{\pi}(z)$ is given remainder of $z\left(z \in \mathbb{Z}_{N(\pi)}\right)$ with respect to modulo $\pi$. We can also consider $z$ as a Lipschitz integer such that its imaginary part is zero. The quotient ring 
of the Lipschitz integers modulo this equivalence relation, which we denote as $\mathcal{L}_{\pi}=\left\{z \bmod \pi: z \in \mathbb{Z}_{N(\pi)}\right\}$. The $\mathcal{L}_{\pi}$ set contains $N(\pi)$ elements. If $\pi$ is a prime Lipschitz integer, then the modulo function $\mu$ defines a bijective mapping from $\mathbb{Z}_{N(\pi)}$ into $\mathcal{L}_{\pi}$ which is a four-dimensional signal space. Therefore, the modulo function $\mu$ is a ring isomorphism between $\mathbb{Z}_{N(\pi)}$ and $\mathcal{L}_{\pi}$. Because there exists a inverse map[8] and we have $\mu\left(z_{1}+z_{2}\right)=\mu\left(z_{1}\right)+\mu\left(z_{2}\right)$ and $\mu\left(z_{1} z_{2}\right)=\mu\left(z_{1}\right) \mu\left(z_{2}\right)$ for any $z_{1}, z_{2} \in \mathbb{Z}_{N(\pi)}$. If $\pi$ is a primitive Lipschitz integer, the modulo function $\mu$ is a group isomorphism with respect to addition between $\mathbb{Z}_{N(\pi)}$ and $\mathcal{L}_{\pi}$. Because there exists a inverse map[8] and we have $\mu\left(z_{1}+z_{2}\right)=\mu\left(z_{1}\right)+\mu\left(z_{2}\right)$ for any $z_{1}, z_{2} \in \mathbb{Z}_{N(\pi)}$. After we define encoder Lipschitz integers set in the following section, we can define a ring isomorphism between $\mathbb{Z}_{N(\pi)}$ and $\mathcal{L}_{\pi}$ where $\pi$ is an encoder Lipschitz integer.

In engineering, the "signal constellation" has been used as a communication term. In mathematics, the "signal constellation" means for residue class set. In the rest of this study, we use "signal constellation" term instead of "left residue class set" term. You can find more details which related to the arithmetics properties of quaternions and Lipschitz integers in [21-22].

\section{Encoder Lipschitz Integers}

The Euclid division algorithm says that there exists unique integers $q$ and $r$ such that $a=b q+r, 0 \leq|r|<|b|$ where $a, b \in \mathbb{Z}$. Here $a$ is the dividend, $b$ is the divisor, $q$ is the quotient, $r$ is the remainder, and $|\cdot|$ is the symbol for absolute value. If we generalize the Euclid division algorithm for Lipschitz integers, then there exists unique Lipschitz integers $\beta$ and $\gamma$ such that $\alpha=\pi \beta+\gamma, 0 \leq N(\gamma) \leq N(\pi)$ where $\alpha, \pi \in \mathcal{L}$. Therefore, the Euclid division algorithm does not work for Lipschitz integers because of $0 \leq N(\gamma) \leq N(\pi)$. So, the primitive Lipschitz integers do not have the "division with small remainder" property. The Euclid division algorithm works for prime Lipschitz integers[22]. Because each element in the $\mathcal{L}_{\pi}$ has the minimal norm. So, the prime Lipschitz integers have the "division with small remainder" property. The following proposition and lemma imply that primitive Lipschitz integers do not have the "division with small remainder" property since not working Euclid division algorithm for primitive Lipschitz integers whose each component is an odd integer.

Proposition 3.1. Let $\pi$ is a primitive Lipschitz integer whose each component is an odd integer, and $\frac{N(\pi)}{2} \in \mathbb{Z}_{N(\pi)}$. Then,

$$
N\left(\mu_{\pi}\left(\frac{N(\pi)}{2}\right)\right)=N(\pi)
$$

Proof. Let $\pi=\pi_{1}+\pi_{2} i+\pi_{3} j+\pi_{4} k$ is a Lipschitz integer whose each component is an odd integer such that $\operatorname{gcd}\left(\pi_{1}, \pi_{2}, \pi_{3}, \pi_{4}\right)=1$. The conjugate of 
$\pi$ is $\bar{\pi}=\pi_{1}-\pi_{2} i-\pi_{3} j-\pi_{4} k$. By equation (2.3), we have

$$
\begin{aligned}
\mu_{\pi}\left(\frac{N(\pi)}{2}\right) & =\frac{N(\pi)}{2}-\pi\left\lfloor\frac{\frac{\pi N(\pi)}{2}}{N(\pi)}\right\rceil \\
& =\frac{N(\pi)}{2}-\pi\left\lfloor\frac{\pi}{2}\right\rceil \\
& =\frac{N(\pi)}{2}-\left(\pi_{1}+\pi_{2} i+\pi_{3} j+\pi_{4} k\right)\left\lfloor\frac{\pi_{1}-\pi_{2} i-\pi_{3} j-\pi_{4} k}{2}\right\rceil .
\end{aligned}
$$

$\left\lfloor\frac{\pi_{1}}{2}\right\rceil=\left(\left(\frac{\pi_{1}}{2}\right)+\frac{1}{2}\right),\left\lfloor\frac{-\pi_{2}}{2}\right\rceil=\left(\left(\frac{-\pi_{2}}{2}\right)+\frac{1}{2}\right),\left\lfloor\frac{-\pi_{3}}{2}\right\rceil=\left(\left(\frac{-\pi_{3}}{2}\right)+\frac{1}{2}\right)$ and $\left\lfloor\frac{-\pi_{4}}{2}\right\rceil=$ $\left(\left(\frac{-\pi_{4}}{2}\right)+\frac{1}{2}\right)$ since $\pi_{1}, \pi_{2}, \pi_{3}$ and $\pi_{4}$ are odd integers. Then,

$$
\begin{aligned}
\mu_{\pi}\left(\frac{N(\pi)}{2}\right)= & \frac{N(\pi)}{2}-\left(\pi_{1}+\pi_{2} i+\pi_{3} j+\pi_{4} k\right)\left[\left(\frac{\pi_{1}}{2}+\frac{1}{2}\right)+\left(\frac{-\pi_{2}}{2}+\frac{1}{2}\right) i\right. \\
& \left.+\left(\frac{-\pi_{3}}{2}+\frac{1}{2}\right) j+\left(-\frac{\pi_{4}}{2}+\frac{1}{2}\right) k\right] \\
= & \frac{N(\pi)}{2}-\left(\frac{\pi_{1}^{2}+\pi_{1}}{2}+\left(\frac{-\pi_{1} \pi_{2}+\pi_{1}}{2}\right) i+\left(\frac{-\pi_{1} \pi_{3}+\pi_{1}}{2}\right) j\right. \\
& +\left(\frac{-\pi_{1} \pi_{4}+\pi_{1}}{2}\right) k+\left(\frac{\pi_{2} \pi_{1}+\pi_{2}}{2}\right) i+\frac{\pi_{2}^{2}-\pi_{2}}{2}+\left(\frac{-\pi_{2} \pi_{3}+\pi_{2}}{2}\right) k \\
& +\left(\frac{\pi_{2} \pi_{4}-\pi_{2}}{2}\right) j+\left(\frac{\pi_{3} \pi_{1}+\pi_{3}}{2}\right) j+\left(\frac{\pi_{3} \pi_{2}-\pi_{3}}{2}\right) k+\left(\frac{\pi_{3}^{2}-\pi 3}{2}\right) \\
& +\left(\frac{-\pi_{3} \pi_{4}+\pi_{3}}{2}\right) i+\left(\frac{\pi_{4} \pi_{1}+\pi_{4}}{2}\right) k+\left(\frac{-\pi_{4} \pi_{2}+\pi_{4}}{2}\right) j \\
& +\left(\frac{\pi_{4} \pi_{3}-\pi_{4}}{2}\right) i+\frac{\pi_{4}^{2}-\pi_{4}}{2} \\
= & \frac{N(\pi)}{2}-\left[\frac{\pi_{1}^{2}+\pi_{2}^{2}+\pi_{3}^{2}+\pi_{4}^{2}+\pi_{1}-\pi_{2}-\pi_{3}-\pi_{4}}{2}+\left(\frac{-\pi_{1} \pi_{2}+\pi_{1}+\pi_{2} \pi_{1}+\pi_{2}-\pi_{3} \pi_{4}+\pi_{3}+\pi_{4} \pi_{3}-\pi_{4}}{2}\right) i\right. \\
& +\left(\frac{-\pi_{1} \pi_{3}+\pi_{1}+\pi_{2} \pi_{4}-\pi_{2}+\pi_{3} \pi_{1}+\pi_{3}-\pi_{4} \pi_{2}+\pi_{4}}{2}\right) j \\
& \left.+\left(\frac{-\pi_{1} \pi_{4}+\pi_{1}-\pi_{2} \pi_{3}+\pi_{2}+\pi_{3} \pi_{2}-\pi_{3}+\pi_{4} \pi_{1}+\pi_{4}}{2}\right) k\right] \\
= & \frac{N(\pi)}{2}-\frac{N(\pi)}{2}-\frac{\pi_{1}-\pi_{2}-\pi_{3}-\pi_{4}}{2}+\left(\frac{\pi_{1}+\pi_{2}+\pi_{3}-\pi_{4}}{2}\right) i \\
& +\left(\frac{\pi_{1}-\pi_{2}+\pi_{3}+\pi_{4}}{2}\right) j+\left(\frac{\pi_{1}+\pi_{2}-\pi_{3}+\pi_{4}}{2}\right) k \\
= & -\frac{\pi_{1}-\pi_{2}-\pi_{3}-\pi_{4}}{2}+\left(\frac{\pi_{1}+\pi_{2}+\pi_{3}-\pi_{4}}{2}\right) i+\left(\frac{\pi_{1}-\pi_{2}+\pi_{3}+\pi_{4}}{2}\right) j \\
& +\left(\frac{\pi_{1}+\pi_{2}-\pi_{3}+\pi_{4}}{2}\right) k .
\end{aligned}
$$

Hereby,

$$
\begin{aligned}
N\left(\mu_{\pi}\left(\frac{N(\pi)}{2}\right)\right)= & N\left(-\frac{\pi_{1}-\pi_{2}-\pi_{3}-\pi_{4}}{2}+\left(\frac{\pi_{1}+\pi_{2}+\pi_{3}-\pi_{4}}{2}\right) i\right. \\
& \left.+\left(\frac{\pi_{1}-\pi_{2}+\pi_{3}+\pi_{4}}{2}\right) j+\left(\frac{\pi_{1}+\pi_{2}-\pi_{3}+\pi_{4}}{2}\right) k\right) \\
= & \frac{4\left(\pi_{1}^{2}+\pi_{2}^{2}+\pi_{3}^{2}+\pi_{4}^{2}\right)}{=} \\
= & \pi_{1}^{2}+\pi_{2}^{2}+\pi_{3}^{2}+\pi_{4}^{2} .
\end{aligned}
$$

Since $N(\pi)=\pi_{1}^{2}+\pi_{2}^{2}+\pi_{3}^{2}+\pi_{4}^{2}$, we have

$$
N\left(\mu_{\pi}\left(\frac{N(\pi)}{2}\right)\right)=N(\pi)
$$

This completes the proof.

Let $\gamma=\mu_{\pi}\left(\frac{N(\pi)}{2}\right)$. By proposition 3.1, we have $N\left(\mu_{\pi}\left(\frac{N(\pi)}{2}\right)\right)=N(\gamma)=$ $N(\pi)$. In other words, the norm of the remainder is equal to the norm of the divisor. We generalize proposition 3.1 for primitive Lipschitz integers whose norm is not a prime integer with the following lemma.

Lemma 3.1. Let $\pi$ be a primitive Lipschitz integer whose norm is not a prime integer. Then,

$$
N\left(\mu_{\pi}(\alpha)\right) \leq N(\pi)
$$

where $\alpha$ is a Lipschitz integer. 
Proof. Let $\pi$ be a primitive Lipschitz integer, and $\alpha=\alpha_{1}+\alpha_{2} i+\alpha_{3} j+\alpha_{4} k$ be a Lipschitz integers which its norm is non-zero. By equation (2.3), we have

$$
\begin{aligned}
\mu_{\pi}(\alpha) & =\alpha-\pi\left\lfloor\frac{\bar{\pi} \alpha}{N(\pi)}\right\rceil \\
\pi^{-1} \mu_{\pi}(\alpha)= & \pi^{-1} \alpha-\pi^{-1} \pi\left\lfloor\frac{\bar{\pi} \alpha}{\bar{\pi} \pi}\right\rceil \\
= & \pi^{-1} \alpha-\left\lfloor\frac{\alpha}{\pi}\right\rceil \\
= & \pi^{-1}\left(\alpha_{1}+\alpha_{2} i+\alpha_{3} j+\alpha_{4} k\right)-\left\lfloor\frac{\alpha_{1}+\alpha_{2} i+\alpha_{3} j+\alpha_{4} k}{\pi}\right\rceil \\
= & \pi^{-1} \alpha_{1}+\pi^{-1} \alpha_{2} i+\pi^{-1} \alpha_{3} j+\pi^{-1} \alpha_{4} k \\
& -\left\lfloor\pi^{-1} \alpha_{1}+\pi^{-1} \alpha_{2} i+\pi^{-1} \alpha_{3} j+\pi^{-1} \alpha_{4} k\right\rceil \\
= & \pi^{-1} \alpha_{1}+\pi^{-1} \alpha_{2} i+\pi^{-1} \alpha_{3} j+\pi^{-1} \alpha_{4} k \\
& -\left\lfloor\pi^{-1} \alpha_{1}+\pi^{-1} \alpha_{2} i+\pi^{-1} \alpha_{3} j+\pi^{-1} \alpha_{4} k\right\rceil \\
= & \pi^{-1} \alpha_{1}-\left\lfloor\pi^{-1} \alpha_{1}\right\rceil+\left(\pi^{-1} \alpha_{2}-\left\lfloor\pi^{-1} \alpha_{2}\right\rceil\right) i \\
& +\left(\pi^{-1} \alpha_{3}-\left\lfloor\pi^{-1} \alpha_{3}\right\rceil\right) j+\left(\pi^{-1} \alpha_{4}-\left\lfloor\pi^{-1} \alpha_{4}\right\rceil\right) k .
\end{aligned}
$$

Hereby,

$$
\mid \begin{aligned}
& \pi^{-1} \alpha_{1}-\left\lfloor\pi^{-1} \alpha_{1}\right\rceil \mid \leq \frac{1}{2} \\
& \left|\pi^{-1} \alpha_{2}-\left\lfloor\pi^{-1} \alpha_{2}\right\rceil\right| \leq \frac{1}{2} \\
& \left|\pi^{-1} \alpha_{3}-\left\lfloor\pi^{-1} \alpha_{3}\right\rceil\right| \leq \frac{1}{2} \\
& \left|\pi^{-1} \alpha_{4}-\left\lfloor\pi^{-1} \alpha_{4}\right\rceil\right| \leq \frac{1}{2} .
\end{aligned}
$$

So,

$$
\begin{aligned}
& \left(\pi^{-1} \alpha_{1}-\left\lfloor\pi^{-1} \alpha_{1}\right\rceil\right)^{2} \leq\left(\frac{1}{2}\right)^{2} \\
& \left(\pi^{-1} \alpha_{2}-\left\lfloor\pi^{-1} \alpha_{2}\right\rceil\right)^{2} \leq\left(\frac{1}{2}\right)^{2} \\
& \left(\pi^{-1} \alpha_{3}-\left\lfloor\pi^{-1} \alpha_{3}\right\rceil\right)^{2} \leq\left(\frac{1}{2}\right)^{2} \\
& \left(\pi^{-1} \alpha_{4}-\left\lfloor\pi^{-1} \alpha_{4}\right\rceil\right)^{2} \leq\left(\frac{1}{2}\right)^{2} .
\end{aligned}
$$

Consequently,

$$
\begin{aligned}
N\left(\pi^{-1} \mu_{\pi}(\alpha)\right)= & \left(\pi^{-1} \alpha_{1}-\left\lfloor\pi^{-1} \alpha_{1}\right\rceil\right)^{2}+\left(\pi^{-1} \alpha_{2}-\left\lfloor\pi^{-1} \alpha_{2}\right\rceil\right)^{2} \\
& +\left(\pi^{-1} \alpha_{3}-\left\lfloor\pi^{-1} \alpha_{3}\right\rceil\right)^{2}+\left(\pi^{-1} \alpha_{4}-\left\lfloor\pi^{-1} \alpha_{4}\right\rceil\right)^{2} \\
& \leq\left(\frac{1}{2}\right)^{2}+\left(\frac{1}{2}\right)^{2}+\left(\frac{1}{2}\right)^{2}+\left(\frac{1}{2}\right)^{2} \\
N\left(\pi^{-1}\right) N\left(\mu_{\pi}(\alpha)\right) \leq & \frac{1}{4}+\frac{1}{4}+\frac{1}{4}+\frac{1}{4} \\
\frac{1}{N(\pi)} N\left(\mu_{\pi}(\alpha)\right) \leq & 1 \\
N\left(\mu_{\pi}(\alpha)\right) \leq & N(\pi) .
\end{aligned}
$$

This completes this proof.

Example 3.1. Let $\pi=3+i+5 j+3 k$ be a Lipschitz integer. Also, $\pi$ is a primitive Lipschitz integer because of $\operatorname{gcd}(3,1,5,3)=1$. By equation $(2.3)$, 
the Lipschitz constellation is

$$
\mathcal{L}_{\pi}=\left\{\begin{array}{l}
\mu_{\pi}(0)=0, \mu_{\pi}(1)=1, \mu_{\pi}(2)=2, \mu_{\pi}(3)=3, \mu_{\pi}(4)=4, \\
\mu_{\pi}(5)=-3 i+3 j+k, \mu_{\pi}(6)=1-3 i+3 j+k, \\
\mu_{\pi}(7)=2-3 i+3 j+k, \mu_{\pi}(8)=-3+i-3 j+k, \\
\mu_{\pi}(9)=-2+i-3 j+k, \mu_{\pi}(10)=-1+i-3 j+k, \\
\mu_{\pi}(11)=i-3 j+k, \mu_{\pi}(12)=1+i-3 j+k, \\
\mu_{\pi}(13)=2+i-3 j+k, \mu_{\pi}(14)=-2-2 i+2 k, \\
\mu_{\pi}(15)=-1-2 i+2 k, \mu_{\pi}(16)=-2 i+2 k, \\
\mu_{\pi}(17)=-1-2 i+2 k, \mu_{\pi}(18)=2-2 i+2 k, \\
\mu_{\pi}(19)=3-2 i+2 k, \mu_{\pi}(20)=4-2 i+2 k, \\
\mu_{\pi}(21)=5-2 i+2 k, \mu_{\pi}(22)=3-3 i-5 j-k, \\
\mu_{\pi}(23)=-5+2 i-2 k, \mu_{\pi}(24)=-4+2 i-2 k, \\
\mu_{\pi}(25)=-3+2 i-2 k, \mu_{\pi}(26)=-2+2 i-2 k, \\
\mu_{\pi}(27)=-1+2 i-2 k, \mu_{\pi}(28)=2 i-2 k, \\
\mu_{\pi}(29)=1+2 i-2 k, \mu_{\pi}(30)=2+2 i-2 k, \\
\mu_{\pi}(31)=-2-i+3 j-k, \mu_{\pi}(32)=-1-i+3 j-k, \\
\mu_{\pi}(33)=-i+3 j-k, \mu_{\pi}(34)=1-i+3 j-k, \\
\mu_{\pi}(35)=2-i+3 j-k, \mu_{\pi}(36)=3-i+3 j-k, \\
\mu_{\pi}(37)=-2+3 i-3 j-k, \mu_{\pi}(38)=-1+3 i-3 j-k, \\
\mu_{\pi}(39)=3 i-3 j-k, \mu_{\pi}(40)=-4, \mu_{\pi}(41)=-3, \\
\mu_{\pi}(42)=-2, \mu_{\pi}(43)=-1
\end{array}\right\} .
$$

This set contains 44 elements because of $N(\pi)=3^{2}+1^{2}+5^{2}+3^{2}=44$. Also, $N\left(\mu_{\pi}(22)\right)=N(\pi)$ since $N\left(\mu_{\pi}(22)\right)=3^{2}+(-3)^{2}+(-5)^{2}+(-1)^{2}=44$. The norm of other elements in the set (3.11) is less than the norm of $\pi$. So, primitive Lipschitz integer $\pi=3+i+5 j+3 k$ does not have the "division with small remainder" property. In addition, to be a Euclidean metric, the inequality $d\left(x^{\prime}, y^{\prime}\right)+d\left(y^{\prime}, z^{\prime}\right) \geq d\left(x^{\prime}, z^{\prime}\right)$ should be verified. Because the inequalities i) $d\left(x^{\prime}, y^{\prime}\right)=0$ if and only if $x^{\prime}=y^{\prime}$ where $x^{\prime}, y^{\prime} \in \mathcal{L}_{\pi}$, and ii) $d\left(x^{\prime}, y^{\prime}\right)=d\left(y^{\prime}, x^{\prime}\right)$ where $x^{\prime}, y^{\prime} \in \mathcal{L}_{\pi}$, are supplied. We consider $x^{\prime}=3-3 i-5 j-k, y^{\prime}=2$ and $z^{\prime}=1$ in $(3.11) . d\left(x^{\prime}, y^{\prime}\right)=36$ since $N(y-x)=N(2-3+3 i+5 j+k)=N(-1+3 i+5 j+k)=36, d\left(y^{\prime}, z^{\prime}\right)=1$ since $N(z-y)=N(1-2)=N(-1)=1$, and $d\left(x^{\prime}, z^{\prime}\right)=39$ since $N(z-y)=$ $N(1-3+3 i+5 j+k)=N(-2+3 i+5 j+k)=39$. Hereby, we have

$$
\begin{array}{ccc}
d\left(x^{\prime}, y^{\prime}\right)+d\left(y^{\prime}, z^{\prime}\right) & \geq & d\left(x^{\prime}, z^{\prime}\right) \\
36+1 & \geq & 39 \\
37 & \geq & 39 .
\end{array}
$$

But this is not true. Consequently, the Euclidean metric is not provide for the $\mathcal{L}_{\pi}$ constellation constructed by $\pi=3+i+5 j+3 k$ Lipschitz integer, and the Euclidean division algorithm is not work for $\pi=3+i+5 j+3 k$ Lipschitz integer.

We define a set that consists of the primitive Lipschitz integers that have the "division with small remainder" property with the following definition. This set is a subset of the primitive Lipschitz integers, and Lipschitz integers. 
Definition 3.1. Let $\alpha=\alpha_{1}+\alpha_{2} i+\alpha_{3} j+\alpha_{4} k$ be a Lipschitz integer. $\alpha$ Lipschitz integer is called an encoder Lipschitz integer if it is satisfying the following conditions,

- $\alpha_{1}, \alpha_{2}, \alpha_{3}, \alpha_{4} \in \mathbb{Z}^{+}$,

- $\alpha_{1}, \alpha_{2}, \alpha_{3}, \alpha_{4}$ are not same parity, i.e. $\alpha_{1}, \alpha_{2}, \alpha_{3}$ and $\alpha_{4}$ all together neither even integers nor odd integers,

- $\operatorname{gcd}\left(\alpha_{1}, \alpha_{2}, \alpha_{3}, \alpha_{4}\right)=1$.

In other words, a primitive Lipschitz integer whose all components are not odd integers is called an encoder Lipschitz integer. Also, we can check by proposition (3.1) whether a primitive Lipschitz integer is an encoder primitive Lipschitz integer or not.

Let now us show that the modulo function $\mu$ defined between $\mathbb{Z}_{N(\pi)}$ and $\mathcal{L}_{\pi}$ by equation (2.3) is a ring isomorphism with the following theorems.

Theorem 3.1. Let $\pi$ be an encoder Lipschitz integer, and $z_{1}, z_{2} \in \mathbb{Z}_{N(\pi)}$. $\mu: \mathbb{Z}_{N(\pi)} \rightarrow \mathcal{L}_{\pi}$ modulo function is a ring homomorphism with respect to $\mu_{\pi}\left(z_{1}+z_{2}\right)=\mu_{\pi}\left(z_{1}\right)+\mu_{\pi}\left(z_{2}\right) \equiv\left(\mu_{\pi}\left(z_{1}\right)+\mu_{\pi}\left(z_{2}\right)\right) \bmod \pi$ and $\mu_{\pi}\left(z_{1} z_{2}\right)=$ $\mu_{\pi}\left(z_{1}\right) \mu_{\pi}\left(z_{2}\right) \equiv\left(\mu_{\pi}\left(z_{1}\right) \mu_{\pi}\left(z_{2}\right)\right) \bmod \pi$.

Proof. Let $\pi$ be an encoder Lipschitz integer and, $z_{1}, z_{2} \in \mathbb{Z}_{N(\pi)}$. Define $\mu$ : $\mathbb{Z}_{N(\pi)} \rightarrow \mathcal{L}_{\pi}$ modulo function by $\mu_{\pi}(z)=z \bmod \pi=z-\pi\left\lfloor\frac{z \bar{\pi}}{N(\pi)}\right\rceil$. Therefore,

$$
\begin{aligned}
& \mu_{\pi}\left(z_{1}+z_{2}\right)=\left(z_{1}+z_{2}\right) \bmod \pi . \\
& \mu_{\pi}\left(z_{1}+z_{2}\right)=z_{1}+z_{2}-\pi\left\lfloor\frac{\bar{\pi}\left(z_{1}+z_{2}\right)}{N(\pi)}\right\rceil .
\end{aligned}
$$

If $\mu_{\pi}\left(z_{1}\right)=z_{1} \bmod \pi$ and $\mu_{\pi}\left(z_{2}\right)=z_{2} \bmod \pi$, then, there exists $\lambda_{1}$ and $\lambda_{2}$ Lipschitz integers such that $z_{1}=\pi \lambda_{1}+\mu_{\pi}\left(z_{1}\right)$ and $z_{2}=\pi \lambda_{2}+\mu_{\pi}\left(z_{2}\right)$, respectively. Then, we have

$$
\begin{aligned}
\mu_{\pi}\left(z_{1}+z_{2}\right)= & \pi \lambda_{1}+\mu_{\pi}\left(z_{1}\right)+\pi \lambda_{2}+\mu_{\pi}\left(z_{2}\right) \\
& -\pi\left\lfloor\frac{\bar{\pi}\left(\pi \lambda_{1}+\mu_{\pi}\left(z_{1}\right)+\pi \lambda_{2}+\mu_{\pi}\left(z_{2}\right)\right)}{N(\pi)}\right\rceil \\
= & \pi \lambda_{1}+\mu_{\pi}\left(z_{1}\right)+\pi \lambda_{2}+\mu_{\pi}\left(z_{2}\right) \\
& -\pi\left\lfloor\frac{\bar{\pi} \pi \lambda_{1}+\bar{\pi} \mu_{\pi}\left(z_{1}\right)+\bar{\pi} \pi \lambda_{2}+\bar{\pi} \mu_{\pi}\left(z_{2}\right)}{N(\pi)}\right\rceil \\
= & \pi \lambda_{1}+\mu_{\pi}\left(z_{1}\right)+\pi \lambda_{2}+\mu_{\pi}\left(z_{2}\right) \\
& -\pi\left\lfloor\lambda_{1}+\lambda_{2}+\frac{\bar{\pi} \mu_{\pi}\left(z_{1}\right)+\bar{\pi} \mu_{\pi}\left(z_{2}\right)}{N(\pi)}\right\rceil .
\end{aligned}
$$

$\left\lfloor\lambda_{1}+\lambda_{2}\right\rceil=\lambda_{1}+\lambda_{2}$ since $\lambda_{1}+\lambda_{2}$ are Lipschitz integers. Then, we have

$$
\begin{aligned}
\mu_{\pi}\left(z_{1}+z_{2}\right)= & \pi \lambda_{1}+\mu_{\pi}\left(z_{1}\right)+\pi \lambda_{2}+\mu_{\pi}\left(z_{2}\right)-\pi \lambda_{1}-\pi \lambda_{2} \\
& -\pi\left\lfloor+\frac{\bar{\pi} \mu_{\pi}\left(z_{1}\right)+\bar{\pi} \mu_{\pi}\left(z_{2}\right)}{N(\pi)}\right\rceil \\
= & \mu_{\pi}\left(z_{1}\right)+\mu_{\pi}\left(z_{2}\right)-\pi\left\lfloor+\frac{\bar{\pi}\left(\mu_{\pi}\left(z_{1}\right)+\mu_{\pi}\left(z_{2}\right)\right)}{N(\pi)}\right\rceil .
\end{aligned}
$$

So, we have

$$
\mu_{\pi}\left(z_{1}+z_{2}\right)=\left(\mu_{\pi}\left(z_{1}\right)+\mu_{\pi}\left(z_{2}\right)\right) \bmod \pi .
$$

In other words, $\mu_{\pi}\left(z_{1}+z_{2}\right)=\mu_{\pi}\left(z_{1}\right)+\mu_{\pi}\left(z_{2}\right)$. On the other hand,

$$
\begin{aligned}
\mu_{\pi}\left(z_{1} z_{2}\right) & =z_{1} z_{2} \bmod \pi \\
& =z_{1} z_{2}-\pi\left\lfloor\frac{\bar{\pi} z_{1} z_{2}}{N(\pi)}\right\rceil .
\end{aligned}
$$


If $\mu_{\pi}\left(z_{1}\right)=z_{1} \bmod \pi$ and $\mu_{\pi}\left(z_{2}\right)=z_{2} \bmod \pi$, then, there exists $\lambda_{1}$ and $\lambda_{2}$ Lipschitz integers such that $z_{1}=\pi \lambda_{1}+\mu_{\pi}\left(z_{1}\right)$ and $z_{2}=\pi \lambda_{2}+\mu_{\pi}\left(z_{2}\right)$, respectively. Then, we have

$$
\begin{aligned}
\mu_{\pi}\left(z_{1} z_{2}\right)= & \left(\pi \lambda_{1}+\mu_{\pi}\left(z_{1}\right)\right)\left(\pi \lambda_{2}+\mu_{\pi}\left(z_{2}\right)\right) \\
& -\pi\left\lfloor\frac{\bar{\pi}\left(\pi \lambda_{1}+\mu_{\pi}\left(z_{1}\right)\right)\left(\pi \lambda_{2}+\mu_{\pi}\left(z_{2}\right)\right)}{N(\pi)}\right\rceil \\
= & \pi \lambda_{1} \pi \lambda_{2}+\pi \lambda_{1} \mu_{\pi}\left(z_{2}\right)+\mu_{\pi}\left(z_{1}\right) \pi \lambda_{2}+\mu_{\pi}\left(z_{1}\right) \mu_{\pi}\left(z_{2}\right) \\
& -\pi\left\lfloor\frac{\bar{\pi} \pi \lambda_{1} \pi \lambda_{2}+\bar{\pi} \pi \lambda_{1} \mu_{\pi}\left(z_{2}\right)+\bar{\pi} \mu_{\pi}\left(z_{1}\right) \pi \lambda_{2}+\bar{\pi} \mu_{\pi}\left(z_{1}\right) \mu_{\pi}\left(z_{2}\right)}{N(\pi)}\right\rceil \\
= & \pi \lambda_{1} \pi \lambda_{2}+\pi \lambda_{1} \mu_{\pi}\left(z_{2}\right)+\mu_{\pi}\left(z_{1}\right) \pi \lambda_{2}+\mu_{\pi}\left(z_{1}\right) \mu_{\pi}\left(z_{2}\right) \\
& -\pi\left\lfloor\frac{\pi \mu_{\pi}\left(z_{1}\right) \pi \lambda_{2}}{N(\pi)}+\frac{\bar{\pi} \mu_{\pi}\left(z_{1}\right) \mu_{\pi}\left(z_{2}\right)}{N(\pi)}+\lambda_{1} \pi \lambda_{2}+\lambda_{1} \mu_{\pi}\left(z_{2}\right)\right\rceil .
\end{aligned}
$$

So, we have

$$
\mu_{\pi}\left(z_{1} z_{2}\right)=\left(\mu_{\pi}\left(z_{1}\right) \mu_{\pi}\left(z_{2}\right)\right) \bmod \pi .
$$

In other words, $\mu_{\pi}\left(z_{1} z_{2}\right)=\mu_{\pi}\left(z_{1}\right) \mu_{\pi}\left(z_{2}\right)$. Consequently, $\mu$ function is a ring homomorphism. This completes this proof.

Theorem 3.2. Let $\pi$ be an encoder Lipschitz integer. So,

$$
\mathbb{Z}_{N(\pi)} \cong \mathcal{L}_{\pi}
$$

Proof. Let $\pi$ be an encoder Lipschitz integer, and $z \in \mathbb{Z}_{N(\pi)}$. The modulo function defines a mapping from $\mathbb{Z}_{N(\pi)}$ to $\mathcal{L}_{\pi}$. This mapping is $\mu: \mathbb{Z}_{N(\pi)} \rightarrow$ $\mathcal{L}_{\pi}:$

$$
\mu_{\pi}(z)=z \bmod \pi=\gamma=z-\pi\left\lfloor\frac{z \bar{\pi}}{N(\pi)}\right\rceil .
$$

By theorem 3.1, $\mu$ function is a ring homomorphism. This mapping is a surjective ring homomorphism because of $\operatorname{Im} \mu=\left\{\mu_{\pi}(z): z \in \mathbb{Z}_{N(\pi)}\right\}=\mathcal{L}_{\pi}$. If $z=0$ where $z \in \mathbb{Z}_{N(\pi)}$, then

$$
\begin{aligned}
\mu_{\pi}(0) & =0-\pi\left\lfloor\frac{0 \bar{\pi}}{N(\pi)}\right\rceil \\
& =0-\pi\left\lfloor\frac{0 \pi}{N(\pi)}\right\rceil \\
& =0-\pi 0 \\
& =0 .
\end{aligned}
$$

If $z \neq 0$ where $z \in \mathbb{Z}_{N(\pi)}$, then $\mu_{\pi}(z)$ is to greater or equal than 1 . Hereby, this mapping is an injective ring homomorphism because of $\operatorname{Ker} \mu=\{z \in$ $\left.\mathbb{Z}_{N(\pi)}: \mu_{\pi}(z)=0\right\}=\left\{z \in \mathbb{Z}_{N(\pi)}: z=0\right\}=\{0\} \cdot \mu$ function is a ring isomorphism since it is both a surjective ring homomorphism and an injective ring homomorphism, i.e. $\mathbb{Z}_{N(\pi)} \cong \mathcal{L}_{\pi}$. This completes this proof. 
The following theorem demonstrates that the encoder Lipschitz integers have the "division with small remainder" property.

Proposition 3.2. Let $\pi$ is an encoder Lipschitz integer. Then,

$$
N\left(\mu_{\pi}(z)\right)<N(\pi)
$$

Proof. We shall analyze and prove this theorem case by case. Let $\pi=\pi_{1}+$ $\pi_{2} i+\pi_{3} j+\pi_{4} k$ is an encoder Lipschitz integer.

Case : 1 Let $\pi$ is an encoder Lipschitz integer such that $\pi_{1}$ is an even number, and $\pi_{2}, \pi_{3}$ and $\pi_{4}$ are odd integers. So, $N(\pi)$ is an odd integer. By equation $(2.3)$, we have

$$
\mu_{\pi}(z)=z-\pi\left\lfloor\pi^{-1} z\right\rceil
$$

Hereby,

$$
\begin{aligned}
\mu_{\pi}(z) \pi^{-1} & =\pi^{-1} z-\pi^{-1} \pi\left\lfloor\pi^{-1} z\right\rceil \\
& =\pi^{-1} z-\left\lfloor\pi^{-1} z\right\rceil \\
& =\frac{\bar{\pi} z}{N(\pi)}-\left\lfloor\frac{\bar{\pi} z}{N(\pi)}\right\rceil .
\end{aligned}
$$

Since $\bar{\pi}=\pi_{1}-\pi_{2} i-\pi_{3} j-\pi_{4} k$, we have

$$
\begin{aligned}
\mu_{\pi}(z) \pi^{-1}= & \frac{\pi_{1} z}{N(\pi)}-\left(\frac{\pi_{2} z}{N(\pi)}\right) i-\left(\frac{\pi_{3} z}{N(\pi)}\right) j-\left(\frac{\pi_{4} z}{N(\pi)}\right) k \\
& -\left\lfloor\frac{\pi_{1} z}{N(\pi)}\right\rceil-\left\lfloor\frac{\pi_{2} z}{N(\pi)}\right\rceil i-\left\lfloor\frac{\pi_{3} z}{N(\pi)}\right\rceil j-\left\lfloor\frac{\pi_{4} z}{N(\pi)}\right\rceil k \\
= & \frac{\pi_{1} z}{N(\pi)}-\left\lfloor\frac{\pi_{1} z}{N(\pi)}\right\rceil-\left(\frac{\pi_{2} z}{N(\pi)}-\left\lfloor\frac{\pi_{2} z}{N(\pi)}\right\rceil\right) i \\
& -\left(\frac{\pi_{3} z}{N(\pi)}-\left\lfloor\frac{\pi_{3} z}{N(\pi)}\right\rceil\right) j-\left(\frac{\pi_{4} z}{N(\pi)}-\left\lfloor\frac{\pi_{4} z}{N(\pi)}\right\rceil\right) k .
\end{aligned}
$$

Since $\pi_{1}$ is an even integer, $\pi_{2}, \pi_{3}$ and $\pi_{4}$ are odd integers, $N(\pi)$ is an odd integer, and $\frac{N(\pi)}{2}$ is not a integer, we have

$$
\begin{aligned}
& 0 \leq\left|\frac{\pi_{1} z}{N(\pi)}-\left\lfloor\frac{\pi_{1} z}{N(\pi)}\right\rceil\right|<\frac{1}{2} \\
& 0 \leq\left|\frac{\pi_{2} z}{N(\pi)}-\left\lfloor\frac{\pi_{2} z}{N(\pi)}\right\rceil\right|<\frac{1}{2} \\
& 0 \leq\left|\frac{\pi_{3} z}{N(\pi)}-\left\lfloor\frac{\pi_{3} z}{N(\pi)}\right\rceil\right|<\frac{1}{2} \\
& 0 \leq\left|\frac{\pi_{4} z}{N(\pi)}-\left\lfloor\frac{\pi_{4} z}{N(\pi)}\right\rceil\right|<\frac{1}{2} .
\end{aligned}
$$

From equation (3.25),

$$
\begin{aligned}
& N\left(\mu_{\pi}(z) \pi^{-1}\right)=N\left(\frac{\pi_{1} z}{N(\pi)}-\left\lfloor\frac{\pi_{1} z}{N(\pi)}\right\rceil\right)+N\left(\frac{\pi_{2} z}{N(\pi)}-\left\lfloor\frac{\pi_{2} z}{N(\pi)}\right\rceil\right) \\
& +N\left(\frac{\pi_{3} z}{N(\pi)}-\left\lfloor\frac{\pi_{3} z}{N(\pi)}\right\rceil\right)+N\left(\frac{\pi_{4} z}{N(\pi)}-\left\lfloor\frac{\pi_{4} z}{N(\pi)}\right\rceil\right)<\left(\frac{1}{2}\right)^{2}+\left(\frac{1}{2}\right)^{2}+\left(\frac{1}{2}\right)^{2}+\left(\frac{1}{2}\right)^{2}=1 .
\end{aligned}
$$

From equation (3.26),

$$
N\left(\mu_{\pi}(z) \pi^{-1}\right)=N\left(\mu_{\pi}(z)\right) N\left(\pi^{-1}\right)=N\left(\mu_{\pi}(z)\right) \frac{1}{N(\pi)}<1 .
$$

Consequently,

$$
N\left(\mu_{\pi}(z)\right)<N(\pi)
$$

Case : 2 Let $\pi$ is an encoder Lipschitz integer such that $\pi_{1}$ and $\pi_{2}$ are even integers, and $\pi_{3}$ and $\pi_{4}$ are odd integers. Firstly, we should check whether to verify or not proposition 3.1 since $N(\pi)$ is an even integer. Let $z=\frac{N(\pi)}{2}$. By equation (2.3), we have

$$
\mu_{\pi}\left(\frac{N(\pi)}{2}\right)=\frac{N(\pi)}{2}-\pi\left\lfloor\pi^{-1} \frac{N(\pi)}{2}\right\rceil .
$$


Hereby,

$$
\begin{aligned}
\mu_{\pi}\left(\frac{N(\pi)}{2}\right) \pi^{-1} & =\pi^{-1} \frac{N(\pi)}{\frac{2}{\pi}}-\pi^{-1} \pi\left\lfloor\pi^{-1} \frac{N(\pi)}{2}\right\rceil \\
& =\frac{\bar{\pi}}{2}-\left\lfloor\frac{\pi}{2}\right\rceil
\end{aligned}
$$

Since $\bar{\pi}=\pi_{1}-\pi_{2} i-\pi_{3} j-\pi_{4} k$, we have

$$
\begin{aligned}
\mu_{\pi}(z) \pi^{-1}= & \frac{\pi_{1}}{2}-\left(\frac{\pi_{2}}{2}\right) i-\left(\frac{\pi_{3}}{2}\right) j-\left(\frac{\pi_{4}}{2}\right) k \\
& -\left\lfloor\frac{\pi_{1}}{2}\right\rceil+\left\lfloor\frac{\pi_{2}}{2}\right\rceil i+\left\lfloor\frac{\pi_{3}}{2}\right\rceil j+\left\lfloor\frac{\pi_{4}}{2}\right\rceil k \\
= & \frac{\pi_{1}}{2}-\left\lfloor\frac{\pi_{1}}{2}\right\rceil-\left(\frac{\pi_{2}}{2}-\left\lfloor\frac{\pi_{2}}{2}\right\rceil\right) i \\
& -\left(\frac{\pi_{3}}{2}-\left\lfloor\frac{\pi_{3}}{2}\right\rceil\right) j-\left(\frac{\pi_{4}}{2}-\left\lfloor\frac{\pi_{4}}{2}\right\rceil\right) k
\end{aligned}
$$

$\left\lfloor\frac{\pi_{1}}{2}\right\rceil=\frac{\pi_{1}}{2}$ and $\left\lfloor\frac{\pi_{2}}{2}\right\rceil=\frac{\pi_{2}}{2}$ since $\pi_{2}$ and $\pi_{3}$ are even integers. Also, $\left\lfloor\frac{\pi_{3}}{2}\right\rceil=\frac{\pi_{3}+1}{2}$ and $\left\lfloor\frac{\pi_{4}}{2}\right\rceil=\frac{\pi_{4}+1}{2}$ since $\pi_{3}$ and $\pi_{4}$ are odd integers. So,

$$
\begin{aligned}
\mu_{\pi}(z) \pi^{-1}= & \frac{\pi_{1}}{2}-\frac{\pi_{1}}{2}-\left(\frac{\pi_{2}}{2}-\frac{\pi_{2}}{2}\right) i \\
& -\left(\frac{\pi_{3}}{2}-\frac{\pi_{3}+1}{2}\right) j-\left(\frac{\pi_{4}}{2}-\frac{\pi_{4}+1}{2}\right) k \\
= & \left(\frac{1}{2}\right) j+\left(\frac{1}{2}\right) k .
\end{aligned}
$$

Hereby, we have

$$
\begin{array}{ll}
N\left(\mu_{\pi}(z) \pi^{-1}\right) & =N\left(\left(\frac{1}{2}\right) j+\left(\frac{1}{2}\right) k\right) \\
N\left(\mu_{\pi}(z)\right) N\left(\pi^{-1}\right) & =\left(\frac{1}{2}\right)^{2}+\left(\frac{1}{2}\right)^{2} \\
N\left(\mu_{\pi}(z)\right)\left(\frac{1}{N(\pi)}\right) & =\frac{1}{4}+\frac{1}{4} \\
N\left(\mu_{\pi}(z)\right) & =\frac{N(\pi)}{2} .
\end{array}
$$

From equation (3.33),

$$
N\left(\mu_{\pi}(z)\right)<N(\pi) .
$$

Let $z \neq \frac{N(\pi)}{2}$. By equation (2.3), we have

$$
\mu_{\pi}(z)=z-\pi\left\lfloor\pi^{-1} z\right\rceil
$$

Hereby,

$$
\begin{aligned}
\mu_{\pi}(z) \pi^{-1} & =\pi^{-1} z-\pi^{-1} \pi\left\lfloor\pi^{-1} z\right\rceil \\
& =\pi^{-1} z-\left\lfloor\pi^{-1} z\right\rceil \\
& =\frac{\bar{\pi} z}{N(\pi)}-\left\lfloor\frac{\bar{\pi} z}{N(\pi)}\right\rceil .
\end{aligned}
$$

Since $\bar{\pi}=\pi_{1}-\pi_{2} i-\pi_{3} j-\pi_{4} k$, we have

$$
\begin{aligned}
\mu_{\pi}(z) \pi^{-1}= & \frac{\pi_{1} z}{N(\pi)}-\left(\frac{\pi_{2} z}{N(\pi)}\right) i-\left(\frac{\pi_{3} z}{N(\pi)}\right) j-\left(\frac{\pi_{4} z}{N(\pi)}\right) k \\
& -\left\lfloor\frac{\pi_{1} z}{N(\pi)}\right\rceil-\left\lfloor\frac{\pi_{2} z}{N(\pi)}\right\rceil i-\left\lfloor\frac{\pi_{3} z}{N(\pi)}\right\rceil j-\left\lfloor\frac{\pi_{4} z}{N(\pi)}\right\rceil k \\
= & \frac{\pi_{1} z}{N(\pi)}-\left\lfloor\frac{\pi_{1} z}{N(\pi)}-\left(\frac{\pi_{2} z}{N(\pi)}-\left\lfloor\frac{\pi_{2} z}{N(\pi)}\right\rceil\right) i\right. \\
& -\left(\frac{\pi_{3} z}{N(\pi)}-\left\lfloor\frac{\pi_{3} z}{N(\pi)}\right\rceil\right) j-\left(\frac{\pi_{4} z}{N(\pi)}-\left\lfloor\frac{\pi_{4} z}{N(\pi)}\right\rceil\right) k
\end{aligned}
$$

Since $\pi_{1}$ and $\pi_{2}$ are even integers, $\pi_{3}$ and $\pi_{4}$ are odd integers, and $N(\pi)$ is an even integer, we have

$$
\begin{aligned}
& 0 \leq\left|\frac{\pi_{1} z}{N(\pi)}-\left\lfloor\frac{\pi_{1} z}{N(\pi)}\right\rceil\right|<\frac{1}{2} \\
& 0 \leq \mid \frac{\pi_{1} z}{N(\pi)}-\left\lfloor\frac{\pi_{1} z}{N(\pi)}\right\rceil<\frac{1}{2} \\
& 0 \leq \mid \frac{\pi_{3} z}{N(\pi)}-\left\lfloor\frac{\pi_{3} z}{N(\pi)}\right\rceil<\frac{1}{2} \\
& 0 \leq\left|\frac{\pi_{4} z}{N(\pi)}-\left\lfloor\frac{\pi_{4} z}{N(\pi)}\right\rceil\right|<\frac{1}{2} .
\end{aligned}
$$


From equation (3.38),

$$
\begin{aligned}
& N\left(\frac{\pi_{1} z}{N(\pi)}-\left\lfloor\frac{\pi_{1} z}{N(\pi)}\right\rceil\right)+N\left(\frac{\pi_{2} z}{N(\pi)}-\left\lfloor\frac{\pi_{2} z}{N(\pi)}\right\rceil\right)+N\left(\frac{\pi_{3} z}{N(\pi)}-\left\lfloor\frac{\pi_{3} z}{N(\pi)}\right\rceil\right) \\
& +N\left(\frac{\pi_{4} z}{N(\pi)}-\left\lfloor\frac{\pi_{4} z}{N(\pi)}\right\rceil\right)<\left(\frac{1}{2}\right)^{2}+\left(\frac{1}{2}\right)^{2}+\left(\frac{1}{2}\right)^{2}+\left(\frac{1}{2}\right)^{2}=1 .
\end{aligned}
$$

From equation (3.39),

$$
N\left(\mu_{\pi}(z) \pi^{-1}\right)=N\left(\mu_{\pi}(z)\right) N\left(\pi^{-1}\right)=N\left(\mu_{\pi}(z)\right) \frac{1}{N(\pi)}<1 .
$$

Consequently,

$$
N\left(\mu_{\pi}(z)\right)<N(\pi)
$$

Case : 3 Let $\pi$ is an encoder Lipschitz integer such that $\pi_{1}, \pi_{2}$ and $\pi_{3}$ are even integers, and $\pi_{4}$ is an odd integer. So, $N(\pi)$ is an odd integer. By equation (2.3), we have

$$
\mu_{\pi}(z)=z-\pi\left\lfloor\pi^{-1} z\right\rceil
$$

Hereby,

$$
\begin{aligned}
\mu_{\pi}(z) \pi^{-1} & =\pi^{-1} z-\pi^{-1} \pi\left\lfloor\pi^{-1} z\right\rceil \\
& =\pi^{-1} z-\left\lfloor\pi^{-1} z\right\rceil \\
& =\frac{\bar{\pi} z}{N(\pi)}-\left\lfloor\frac{\bar{\pi} z}{N(\pi)}\right\rceil .
\end{aligned}
$$

Since $\bar{\pi}=\pi_{1}-\pi_{2} i-\pi_{3} j-\pi_{4} k$, we have

$$
\begin{aligned}
\mu_{\pi}(z) \pi^{-1}= & \frac{\pi_{1} z}{N(\pi)}-\left(\frac{\pi_{2} z}{N(\pi)}\right) i-\left(\frac{\pi_{3} z}{N(\pi)}\right) j-\left(\frac{\pi_{4} z}{N(\pi)}\right) k \\
& -\left\lfloor\frac{\pi_{1} z}{N(\pi)}\right\rceil-\left\lfloor\frac{\pi_{2} z}{N(\pi)}\right\rceil i-\left\lfloor\frac{\pi_{3} z}{N(\pi)}\right\rceil j-\left\lfloor\frac{\pi_{4} z}{N(\pi)}\right\rceil k \\
= & \frac{\pi_{1} z}{N(\pi)}-\left\lfloor\frac{\pi_{1} z}{N(\pi)}\right\rceil-\left(\frac{\pi_{2} z}{N(\pi)}-\left\lfloor\frac{\pi_{2} z}{N(\pi)}\right\rceil\right) i \\
& -\left(\frac{\pi_{3} z}{N(\pi)}-\left\lfloor\frac{\pi_{3} z}{N(\pi)}\right\rceil\right) j-\left(\frac{\pi_{4} z}{N(\pi)}-\left\lfloor\frac{\pi_{4} z}{N(\pi)}\right\rceil\right) k
\end{aligned}
$$

Since $\pi_{1}, \pi_{2}$ and $\pi_{3}$ are even integers, $\pi_{4}$ is an odd integer, and $N(\pi)$ is an odd integer, we have

$$
\begin{aligned}
& 0 \leq\left|\frac{\pi_{1} z}{N(\pi)}-\left\lfloor\frac{\pi_{1} z}{N(\pi)}\right\rceil\right|<\frac{1}{2} \\
& 0 \leq\left|\frac{\pi_{2} z}{N(\pi)}-\left\lfloor\frac{\pi_{2} z}{N(\pi)}\right\rceil\right|<\frac{1}{2} \\
& 0 \leq\left|\frac{\pi_{3} z}{N(\pi)}-\left\lfloor\frac{\pi_{3} z}{N(\pi)}\right\rceil\right|<\frac{1}{2} \\
& 0 \leq\left|\frac{\pi_{4} z}{N(\pi)}-\left\lfloor\frac{\pi_{4} z}{N(\pi)}\right\rceil\right|<\frac{1}{2} .
\end{aligned}
$$

From equation (3.45),

$$
\begin{aligned}
& N\left(\frac{\pi_{1} z}{N(\pi)}-\left\lfloor\frac{\pi_{1} z}{N(\pi)}\right\rceil\right)+N\left(\frac{\pi_{2} z}{N(\pi)}-\left\lfloor\frac{\pi_{2} z}{N(\pi)}\right\rceil\right)+N\left(\frac{\pi_{3} z}{N(\pi)}-\left\lfloor\frac{\pi_{3} z}{N(\pi)}\right\rceil\right) \\
& +N\left(\frac{\pi_{4} z}{N(\pi)}-\left\lfloor\frac{\pi_{4} z}{N(\pi)}\right\rceil\right)<\left(\frac{1}{2}\right)^{2}+\left(\frac{1}{2}\right)^{2}+\left(\frac{1}{2}\right)^{2}+\left(\frac{1}{2}\right)^{2}=1 .
\end{aligned}
$$

From equation (3.46),

$$
N\left(\mu_{\pi}(z) \pi^{-1}\right)=N\left(\mu_{\pi}(z)\right) N\left(\pi^{-1}\right)=N\left(\mu_{\pi}(z)\right) \frac{1}{N(\pi)}<1 .
$$

Consequently,

$$
N\left(\mu_{\pi}(z)\right)<N(\pi)
$$

This completes this proof.

With the following examples, given an example for each case in the proposition 3.2 . 
Example 3.2. Case $1 \pi=1+3 i+2 j+k$ is an encoder Lipschitz integer. By equation (2.3), the Lipschitz integer constellation is

$$
\mathcal{L}_{\pi}=\left\{\begin{array}{l}
\mu_{\pi}(0)=0, \mu_{\pi}(1)=1, \mu_{\pi}(2)=2, \mu_{\pi}(3)=i+j-2 k, \\
\mu_{\pi}(4)=-1+2 j+k, \mu_{\pi}(5)=2 j+k, \mu_{\pi}(6)=1+2 j+k, \\
\mu_{\pi}(7)=2+2 j+k, \mu_{\pi}(8)=-2-2 j-k, \mu_{\pi}(9)=-1-2 j-k, \\
\mu_{\pi}(10)=-2 j-k, \mu_{\pi}(11)=1-2 j-k, \mu_{\pi}(12)=-i-j+2 k, \\
\mu_{\pi}(13)=-2, \mu_{\pi}(14)=-1,
\end{array}\right\} .
$$

The set contains 15 elements because of $N(\pi)=1^{2}+3^{2}+2^{2}+1^{2}=15$. The norm of each element in the set is less than the norm of $\pi$.

Example 3.3. Case $2 \pi=2+3 i+j+2 k$ is an encoder Lipschitz integer. By equation (2.3), the Lipschitz integer constellation is

$$
\mathcal{L}_{\pi}=\left\{\begin{array}{l}
\mu_{\pi}(0)=0, \mu_{\pi}(1)=1, \mu_{\pi}(2)=2, \mu_{\pi}(3)=3, \\
\mu_{\pi}(4)=1+2 i+2 j-k, \mu_{\pi}(5)=-2-2 j-k, \mu_{\pi}(6)=-1-2 j-k, \\
\mu_{\pi}(7)=-2 j-k, \mu_{\pi}(8)=1-2 j-k, \mu_{\pi}(9)=2-2 j-k, \\
\mu_{\pi}(10)=-1+2 j+k, \mu_{\pi}(11)=2 j+k, \mu_{\pi}(12)=1+2 j+k, \\
\mu_{\pi}(13)=2+2 j+k, \mu_{\pi}(14)=-1-2 i-2 j+k, \\
\mu_{\pi}(15)=-2 i-2 j+k, \mu_{\pi}(16)=-2, \mu_{\pi}(17)=-1
\end{array}\right\} .
$$

The set contains 18 elements because of $N(\pi)=2^{2}+3^{2}+1^{2}+2^{2}=18$. The norm of each element in the set is less than the norm of $\pi$.

Example 3.4. Case $3 \pi=2+3 i+2 j+2 k$ is an encoder Lipschitz integer. By equation (2.3), the Lipschitz integer constellation is

$$
\mathcal{L}_{\pi}=\left\{\begin{array}{l}
\mu_{\pi}(0)=0, \mu_{\pi}(1)=1, \mu_{\pi}(2)=2, \mu_{\pi}(3)=3, \\
\mu_{\pi}(4)=1+2 i+2 j-2 k, \mu_{\pi}(5)=2+2 i+2 j-2 k, \\
\mu_{\pi}(6)=3-i-j+k, \mu_{\pi}(7)=-2-i-j+k, \\
\mu_{\pi}(8)=-1-i-j+k, \mu_{\pi}(9)=-i-j+k, \\
\mu_{\pi}(10)=1-i-j+k, \mu_{\pi}(11)=-1+i+j-k, \\
\mu_{\pi}(12)=i+j-k, \mu_{\pi}(13)=1+i+j-k, \\
\mu_{\pi}(14)=2+i+j-k, \mu_{\pi}(15)=3+i+j-k, \\
\mu_{\pi}(16)=-2-2 i-2 j+2 k, \mu_{\pi}(17)=-1-2 i-2 j+2 k, \\
\mu_{\pi}(18)=-3, \mu_{\pi}(19)=-2, \mu_{\pi}(17)=-1
\end{array}\right\} .
$$

The set contains 21 elements because of $N(\pi)=2^{2}+3^{2}+2^{2}+2^{2}=21$. The norm of each element in the set is less than the norm of $\pi$.

Example 3.2, example 3.3, and example 3.4 are verified all the conditions for it to be an Euclidean metric. Also the Euclidean division algorithm works for the Lipschitz integers in example 3.2, example 3.3, and example 3.4. As a result of these examples, we represent the following proposition.

Proposition 3.3. Let $\pi$ be an encoder Lipschitz integer. Then,

$$
\mu_{\pi}(\alpha)+\mu_{\pi}(N(\pi)-\alpha)=0
$$

where $\alpha$ is a Lipschitz integer, and

$$
\mu_{\pi}(z)+\mu_{\pi}(N(\pi)-z)=0
$$

where $z \in \mathbb{Z}_{N(\pi)}$. 
Proof. Firstly, let show us that $\mu_{\pi}(\alpha)+\mu_{\pi}(N(\pi)-\alpha)=0$ where $\pi$ is an encoder Lipschitz integer, and $\alpha$ is a Lipschitz integer. By equation (2.3), we have

$$
\begin{aligned}
\mu_{\pi}(\alpha)+\mu_{\pi}(N(\pi)-\alpha) & =\alpha-\pi\left\lfloor\frac{\alpha \bar{\pi}}{N(\pi)}\right\rceil N(\pi)-\alpha-\pi\left\lfloor\frac{(N(\pi)-\alpha) \bar{\pi}}{N(\pi)}\right\rceil \\
& =N(\pi)-\pi\left\lfloor\frac{\alpha \bar{\pi}}{N(\pi)}\right\rceil-\pi\left\lfloor\frac{\bar{\pi} N(\pi)}{N(\pi)}-\frac{\alpha \bar{\pi}}{N(\pi)}\right\rceil \\
& =N(\pi)-\pi\left\lfloor\frac{\alpha \bar{\pi}}{N(\pi)}\right\rceil-\pi\left\lfloor\bar{\pi}-\frac{\alpha \bar{\pi}}{N(\pi)}\right\rceil .
\end{aligned}
$$

$\left\lfloor\bar{\pi}-\frac{\alpha \bar{\pi}}{N(\pi)}\right\rceil=\lfloor\bar{\pi}\rceil-\left\lfloor\frac{\alpha \bar{\pi}}{N(\pi)}\right\rceil=\bar{\pi}-\left\lfloor\frac{\alpha \bar{\pi}}{N(\pi)}\right\rceil$ since $\bar{\pi}$ is an encoder Lipschitz integer. Consequently,

$$
\begin{aligned}
\mu_{\pi}(\alpha)+\mu_{\pi}(N(\pi)-\alpha) & =N(\pi)-\pi\left\lfloor\frac{\alpha \bar{\pi}}{N(\pi)}\right\rceil-\pi \bar{\pi}+\pi\left\lfloor\frac{\alpha \bar{\pi}}{N(\pi)}\right\rceil \\
& =N(\pi)-N(\pi) \\
& =0 .
\end{aligned}
$$

Now, let show us that $\mu_{\pi}(z)+\mu_{\pi}(N(\pi)-z)=0$ where $\pi$ is an encoder Lipschitz integer and, $z \in \mathbb{Z}_{N(\pi)}$. By equation (2.3), we have

$$
\begin{aligned}
\mu_{\pi}(z)+\mu_{\pi}(N(\pi)-z) & =z-\pi\left\lfloor\frac{z \bar{\pi}}{N(\pi)}\right\rceil+N(\pi)-z-\pi\left\lfloor\frac{(N(\pi)-z) \bar{\pi}}{N(\pi)}\right\rceil \\
& =N(\pi)-\pi\left\lfloor\frac{z \bar{\pi}}{N(\pi)}\right\rceil-\pi\left\lfloor\frac{\bar{\pi} N(\pi)}{N(\pi)}-\frac{z \bar{\pi}}{N(\pi)}\right\rceil \\
& =N(\pi)-\pi\left\lfloor\frac{z \bar{\pi}}{N(\pi)}\right\rceil-\pi\left\lfloor\pi-\frac{z \bar{\pi}}{N(\pi)}\right\rceil .
\end{aligned}
$$

$\left\lfloor\bar{\pi}-\frac{z \bar{\pi}}{N(\pi)}\right\rceil=\lfloor\bar{\pi}\rceil-\left\lfloor\frac{z \bar{\pi}}{N(\pi)}\right\rceil=\bar{\pi}-\left\lfloor\frac{z \bar{\pi}}{N(\pi)}\right\rceil$ since $\bar{\pi}$ is an encoder Lipschitz integer. Consequently,

$$
\begin{aligned}
\mu_{\pi}(z)+\mu_{\pi}(N(\pi)-z) & =N(\pi)-\pi\left\lfloor\frac{z \bar{\pi}}{N(\pi)}\right\rceil-\pi \bar{\pi}+\pi\left\lfloor\frac{z \bar{\pi}}{N(\pi)}\right\rceil \\
& =N(\pi)-N(\pi) \\
& =0 .
\end{aligned}
$$

\section{Performances of Lipschitz Constellations for Transmission over AWGN channel}

In this section, we are first giving some distance and performance measures, and then we investigate the performance of Lipschitz constellations that lies on encoder Lipschitz integers for transmission over AWGN channel by agency of average energy, CFM, and SNR gains. Also, we give set partitioning property on larger Lipschitz integers,namely proposed Lipschitz integers. You can found more further information in [14-15]. We follow the procedures in [15] for some distance, performance measures and set partitioning property.. The average energy of a constellation denoted by $\mathcal{E}_{\pi}$ is computed by

$$
\mathcal{E}_{\pi}=\frac{1}{N(\pi)} \sum_{z=0}^{N(\pi)-1} N\left(\mu_{\pi}(z)\right) .
$$

The squared Euclidean distance of two Lipschitz integers is defined as

$$
d_{E}(\alpha, \beta)=N(\beta-\alpha)
$$


and the minimum squared Euclidean distance of the constellation is

$$
\delta_{\lambda}^{2}=\min _{\alpha \neq \beta} d_{E}(\alpha, \beta)
$$

where $\alpha, \beta \in \mathcal{L}_{\pi}$. In [23], Forney and Wei proposed the constellation figure of merit (CFM) to compare signal constellations of different dimensions. The $\mathrm{CFM}$ is the ratio of the minimum squared Euclidean distance and the average energy per two-dimensions. So, the CFM of a $M$-dimensional constellation is computed by

$$
C F M=\frac{M \delta_{\pi}^{2}}{2 \mathcal{E}_{\pi}}
$$

A higher CFM leads to a better performance for transmission over an AWGN channel. Asymptotic coding gain means for higher signal to noise ratio (SNR) [9]. The SNR of $M$-dimensional constellation is computed by

$$
S N R=-10 \cdot \log _{10}(\mathrm{CFM} \text { of signal constellation }) .
$$

The SNR gains of a Lipschitz constellation over the AWGN channel is computed by

$$
S N R=-10 \cdot \log _{10}\left(\frac{\mathrm{CFM} \text { of } \mathcal{L}_{\pi}}{\mathrm{CFM} \text { of Gaussian constellation }}\right)
$$

where Lipschitz constellation $\mathcal{L}_{\pi}$ and Gaussian constellation are the equal size. A residue class ring of Lipschitz integers $\mathcal{L}_{\pi}$ arises from the residue class ring of integers $\mathbb{Z}_{N(\pi)}=\{0,1, \ldots, N(\pi)-1\}$ for an integer $N(\pi)$. If $N(\pi)$ is not a prime integer, then we can partition the set $\mathbb{Z}_{N(\pi)}$ into subsets of equal size. Let $N=c \cdot d$ where $N$ is the elements number of Lipschitz constellation. We can partition the set $\mathcal{L}_{\pi}$ into c subsets $\mathcal{L}_{\pi}^{(0)}, \ldots, \mathcal{L}_{\pi}^{(c-1)}$ each with $d$ elements. The subsets correspond to the integer sets $\mathbb{Z}_{\pi}^{(0)}, \ldots, \mathbb{Z}_{\pi}^{(c-1)}$, where

$$
\mathbb{Z}_{N(\pi)}^{(0)}=\{0, c, 2 c, \ldots,(d-1) c\}
$$

and $\mathbb{Z}_{\pi}^{(1)}, \ldots, \mathbb{Z}_{\pi}^{(c-1)}$ are the cosets of $\mathbb{Z}_{\pi}^{(0)}$, i.e. $\mathbb{Z}_{\pi}^{(l)}=\left\{z: z-l \in \mathbb{Z}_{\pi}^{(0)}\right\}$. Note that the number of elements of the Lipschitz constellation and Gaussian constellation should be the equal size to compare performances over the AWGN channel but proposed Lipschitz constellations should not be. Hence, we can apply set partitioning property on proposed Lipschitz constellations. The SNR gains of a proposed Lipschitz constellation over the AWGN channel is computed by 


$$
\begin{aligned}
S N R= & -10 \cdot \log _{10}\left(\frac{\mathrm{CFM} \text { of } \mathcal{L}_{\pi}^{(0)}}{\mathrm{CFM} \text { of Gaussian constellation }}\right) \\
& +10 \cdot \log _{10}\left(\frac{\mathrm{CFM} \text { of Lipschitz constellation }}{\mathrm{CFM} \text { of Gaussian constellation }}\right) \\
= & -10\left(\log _{10}\left(\mathrm{CFM} \text { of } \mathcal{L}_{\pi}^{(0)}\right)\right. \\
& \left.-\log _{10}(\mathrm{CFM} \text { of Lipschitz constellation })\right) \\
& +10 \cdot \log _{10}(\mathrm{CFM} \text { of Gaussian constellation }) \\
& -10 \cdot \log _{10}(\mathrm{CFM} \text { of Gaussian constellation }) \\
= & -10\left(\log _{10}\left(\mathrm{CFM} \text { of } \mathcal{L}_{\pi}^{(0)}\right)\right. \\
& \left.-\log _{10}(\mathrm{CFM} \text { of Lipschitz constellation })\right) \\
= & -10 \log _{10}\left(\frac{\mathrm{CFM} \text { of } \mathcal{L}_{\pi}^{(0)}}{\mathrm{CFM} \text { of Lipschitz constellation }}\right)
\end{aligned}
$$

where Lipschitz signal constellation $\mathcal{L}_{\pi}^{(0)}$ and the Lipschitz constellation are the equal size. In [15],[24], Freudenberger et al. investigated Lipschitz signal constellations according to their performance for transmission over the AWGN channel by the agency of CFM. In [24], Freudenberger et al. presented a comparison of all Gaussian and Lipschitz signal constellations of the same size for $N(\pi)<100$, where both signal constellations exist. They demonstrated that both signal constellations have almost same CFM. Thereby they concluded that these Lipschitz signal constellations have no performance advantage for transmission over an AWGN channel. To Lipschitz signal constellations have performance advantage for transmission over an AWGN channel, they constructed new Lipschitz signal constellations based on set partitioning of larger signal sets. In [15], Freudenberger et al. investigated proposed Lipschitz signal constellations that have set partitioning property according to their performance for transmission over the AWGN channel by the agency of CFM. We present different proposed primitive Lipschitz integers that have the "division with small remainder" in Table I instead of the proposed primitive Lipschitz integers that do not have the "division with small remainder" property used to constructed proposed Lipschitz constellations in [15, Table I]. You can examine the below example 4.1 and example 4.2.

Example 4.1. In [15, Table I], Freudenberger et al. presented $9+7 i+5 j+k$ proposed primitive Lipschitz integer with $N=c \cdot d=6 \cdot 26=156$. They gave CFM and SNR gain of proposed Lipschitz constellation that has 156 elements with respect to primitive Lipschitz constellation with 26 elements, which is a subset that has a larger minimum square Euclidean distance of proposed Lipschitz constellation with 156 elements. But proposed primitive Lipschitz integer with 156 elements does not has the "division with small remainder" property since the Euclid division algorithm does not work with respect to proposition 3.1. Also, all proposed primitive Lipschitz integers with $N=$ $c \cdot d=6 \cdot 26=156$ do not have the "division with small remainder" property. These proposed primitive Lipschitz integers with $N=c \cdot d=6 \cdot 26=156$ are $7+7 i+7 j+3 k, 9+5 i+5 j+5 k, 9+7 i+5 j+k$, and $11+5 i+3 j+k$. So, instead of $9+7 i+5 j+k$ proposed primitive Lipschitz integer, we propose $10+5 i+2 j+k$ proposed primitive Lipschitz integer with $N=c \cdot d=$ 
$5 \cdot 26=130$. This proposed primitive Lipschitz integer has the "division with small remainder" property since it will be verified by proposition 3.2. Shortly, we propose $10+5 i+2 j+k$ encoder Lipschitz integer in Table I instead of the proposed primitive Lipschitz integer that is given for $d=26$ since the proposed primitive Lipschitz integer that is given for $d=26$ in [15, Table I] does not has the "division with small remainder" property. We present CFM and SNR gain of this proposed Lipschitz constellation with respect to primitive Lipschitz constellation with 26 elements, which is a subset that has a larger minimum square Euclidean distance of this proposed Lipschitz constellation in Table I.

Example 4.2. In [15, Table I], Freudenberger et al. presented $19+17 i+9 j+3 k$ proposed primitive Lipschitz integer with $N=c \cdot d=10 \cdot 74=740$. They gave CFM and SNR gain of this proposed Lipschitz constellation with respect to primitive Lipschitz constellation with 74 elements, which is a subset that has a larger minimum square Euclidean distance of this proposed Lipschitz constellation. But this proposed primitive Lipschitz integer does not has the "division with small remainder" property since the Euclid division algorithm does not work with respect to proposition 3.1. Also, all proposed primitive Lipschitz integers with $N=c \cdot d=10 \cdot 74=740$ do not have the "division with small remainder" property. These proposed primitive Lipschitz integers with $N=c \cdot d=10 \cdot 74=740$ are $15+15 i+13 j+11 k, 17+15 i+15 j+k$, $17+17 i+9 j+9 k, 19+19 i+3 j+3 k, 21+13 i+9 j+7 k, 21+13 i+11 j+3 k$, $21+15 i+7 j+5 k, 21+17 i+3 j+k, 23+9 i+9 j+7 k, 23+11 i+9 j 3 k$, $25+9 i+5 j+3 k$ and $27+3 i+j+k$. So, instead of $19+17 i+9 j+3 k$ proposed primitive Lipschitz integer, we propose $17+14 i+10 j+9 k$ proposed primitive Lipschitz integer with $N=c \cdot d=9 \cdot 74=666$. This proposed primitive Lipschitz integer has the "division with small remainder" property since it will be verified by proposition 3.2. The Euclid division algorithm works for this encoder Lipschitz integer. We present CFM and SNR gain of this proposed Lipschitz constellation with respect to primitive Lipschitz constellation with 26 elements, which is a subset that has a larger minimum square Euclidean distance of this proposed Lipschitz constellation in Table I. You can see Table II for other proposed primitive Lipschitz integers with $N=c \cdot d=9 \cdot 74=666$.

Example 4.3. In Table II, we present other proposed primitive Lipschitz integers used to construct proposed Lipschitz constellations that have higher CFM for some $N$ values that have set partitioning property. These proposed primitive Lipschitz integers have the "division with small remainder" property since it will be verified by proposition 3.2. We do not present proposed Lipschitz integers used to construct proposed primitive Lipschitz constellations with $d=10,13,25,26,34,65$, or 89 elements in this paper since there exists just one. You can see $[15$, Table I] for them.

Example 4.4. There also exist different primitive Lipschitz integers used to construct Lipschitz constellations that have higher CFM and lower average 
TABLE 1. Table of CFM, Average Energy and, SNR coding gain of proposed primitive Lipschitz constellations for $\mathrm{d}=26$ and $\mathrm{d}=74(\mathrm{~N}$ : The number of elements (norm) of a proposed primitive Lipschitz signal constellation, c: The number of subsets of a proposed primitive Lipschitz signal constellation, d:The number of elements (norm) of subset of a proposed primitive Lipschitz signal constellation )

\begin{tabular}{|c|c|c|l|l|l|l|l|l|}
\hline & & & & \multicolumn{3}{|c|}{ Constellations } & CFM & $\begin{array}{l}\text { Average } \\
\text { Energy of } \\
\text { Proposed } \\
\text { N }\end{array}$ \\
\cline { 5 - 8 } & & $\mathrm{c}$ & $\mathrm{d}$ & $\begin{array}{l}\text { Proposed } \\
\text { Primitive } \\
\text { Lipschitz } \\
\text { Integers }\end{array}$ & Gauss & Lipschitz & $\begin{array}{l}\text { Proposed } \\
\text { Lipschitz }\end{array}$ & $\begin{array}{l}\text { SNR } \\
\text { gain } \\
\text { [dB] }\end{array}$ \\
\hline 130 & 5 & 26 & $10+5 \mathrm{i}+2 \mathrm{j}+\mathrm{k}$ & 0.2301 & 0.2119 & 1.1556 & 43.2692 & 7.37 \\
\hline 666 & 9 & 74 & $17+14 \mathrm{i}+10 \mathrm{j}+9 \mathrm{k}$ & 0.0811 & 0.0811 & 0.7299 & 221.9590 & 9.54 \\
\hline
\end{tabular}

TABLE 2. Table CFM and SNR coding gain of some proposed Lipschitz constellations ( $\mathrm{N}$ : The number of elements (norm) of a proposed Lipschitz signal constellation, c: The number of subsets of a proposed Lipschitz signal constellation, d:The number of elements (norm) of subset of a proposed Lipschitz signal constellation )

\begin{tabular}{|c|c|c|l|l|l|l|l|l|}
\hline & & & & \multicolumn{3}{|c|}{ Constellations } & CFM & Average \\
Energy of \\
Proposed \\
N
\end{tabular}

energy in equal size. You can see Table II and Table III. In Table III, we present all primitive Lipschitz integers used to construct Lipschitz constellations with $d<100$ elements, and CFM, average energy, and SNR gain of Lipschitz constellations that have same size with Gaussian constellations.

The below examples are given clues about the construction of tables.

Example 4.5. We consider proposed Lipschitz constellation(s) with $N=$ $3 \cdot 13=39$ elements. There exist two different proposed primitive Lipschitz integers used to construct proposed Lipschitz constellations with $N=39$. These proposed primitive Lipschitz integers are $5+3 i+2 j+k$ and $6+i+j+k$. There 
TABLE 3. Table of CFM, Energy and SNR gain of all possible primitive Lipschitz constellation that have higher CFM and have minimum Energy for $d \leq 100$ ( $d$ :The number of elements (norm) of a primitive Lipschitz signal constellation, $\mathcal{E}_{s}$ : Average Energy )

\begin{tabular}{|c|c|c|c|c|c|}
\hline \multirow[t]{2}{*}{$\mathrm{d}$} & \multirow{2}{*}{$\begin{array}{l}\text { Lipschitz } \\
\text { Integers }\end{array}$} & \multicolumn{2}{|c|}{$\begin{array}{c}\text { Lipschitz } \\
\text { Constellation }\end{array}$} & \multirow{2}{*}{$\begin{array}{l}\text { CFM of Gauss } \\
\text { Constellation }\end{array}$} & \multirow{2}{*}{$\begin{array}{c}\text { SNR } \\
\text { Gain }[\mathrm{dB}]\end{array}$} \\
\hline & & $\mathcal{E}_{s}$ & CFM & & \\
\hline 10 & $2+2 \mathrm{i}+\mathrm{j}+\mathrm{k}$ & 3.3000 & 0.6061 & 0.5882 & 0.13 \\
\hline 13 & $2+2 \mathrm{i}+2 \mathrm{j}+\mathrm{k}$ & 4.3077 & 0.4643 & 0.4643 & 0 \\
\hline 25 & $4+2 \mathrm{i}+2 \mathrm{j}+\mathrm{k}$ & 8.3200 & 0.2404 & 0.2404 & 0 \\
\hline 26 & $3+3 i+2 j+2 k$ & 8.6539 & 0.2311 & 0.2301 & 0.02 \\
\hline 34 & $\begin{array}{c}4+4 \mathrm{i}+\mathrm{j}+\mathrm{k} \\
5+2 \mathrm{i}+2 \mathrm{j}+\mathrm{k}\end{array}$ & 11.3235 & 0.1766 & 0.1762 & 0.01 \\
\hline 37 & $\begin{array}{c}4+4 i+2 j+k \\
5+2 i+2 j+2 k\end{array}$ & 12.3243 & 0.1623 & 0.1623 & 0 \\
\hline 50 & $\begin{array}{c}4+4 i+3 j+3 k \\
6+3 i+2 j+k\end{array}$ & 16.6600 & 0.1200 & 0.1199 & 0 \\
\hline 53 & $6+3 i+2 j+2 k$ & 17.6604 & 0.1132 & 0.1132 & 0 \\
\hline 58 & $\begin{array}{c}5+4 \mathrm{i}+4 \mathrm{j}+\mathrm{k} \\
5+5 \mathrm{i}+2 \mathrm{j}+2 \mathrm{k} \\
6+3 \mathrm{i}+3 \mathrm{j}+2 \mathrm{k} \\
7+2 \mathrm{i}+2 \mathrm{j}+\mathrm{k}\end{array}$ & 19.3276 & 0.1035 & 0.1034 & 0 \\
\hline 61 & $\begin{array}{c}5+4 \mathrm{i}+4 \mathrm{j}+2 \mathrm{k} \\
7+2 \mathrm{i}+2 \mathrm{j}+\mathrm{k}\end{array}$ & 20.3279 & 0.0984 & 0.0984 & 0 \\
\hline 65 & $6+4 i+3 j+2 k$ & 21.6615 & 0.0923 & 0.0923 & 0 \\
\hline 73 & $\begin{array}{c}5+4 \mathrm{i}+4 \mathrm{j}+4 \mathrm{k} \\
7+4 \mathrm{i}+2 \mathrm{j}+2 \mathrm{k} \\
8+2 \mathrm{i}+2 \mathrm{j}+\mathrm{k}\end{array}$ & 24.3288 & 0.0822 & 0.0822 & 0 \\
\hline 74 & $\begin{array}{c}6+5 i+3 j+2 k \\
6+6 i+j+k\end{array}$ & 24.6622 & 0.0811 & 0.0811 & 0 \\
\hline 82 & $\begin{array}{c}5+5 \mathrm{i}+4 \mathrm{j}+4 \mathrm{k} \\
6+6 \mathrm{i}+3 \mathrm{j}+\mathrm{k} \\
7+4 \mathrm{i}+4 \mathrm{j}+\mathrm{k} \\
7+5 \mathrm{i}+2 \mathrm{j}+2 \mathrm{k} \\
8+4 \mathrm{i}+\mathrm{j}+\mathrm{k}\end{array}$ & 27.3293 & 0.0732 & 0.0731 & 0 \\
\hline 85 & $\begin{array}{c}6+6 i+3 j+2 k \\
7+4 i+4 j+2 k \\
8+4 i+2 j+k\end{array}$ & 28.3294 & 0.0706 & 0.0706 & 0 \\
\hline 89 & $6+4 i+3 j+2 k$ & 29.6629 & 0.0674 & 0.0674 & 0 \\
\hline 97 & $\begin{array}{c}6+6 i+4 j+3 k \\
7+4 i+4 j+4 k \\
8+5 i+2 j+2 k \\
8+4 i+4 j+k\end{array}$ & 32.3299 & 0.0619 & 0.0619 & 0 \\
\hline
\end{tabular}


is no Gaussian constellation that has an equal size with the proposed Lipschitz constellation that has $N=39$ elements. Note that proposed primitive Lipschitz integers are not to be the same size as primitive Gaussian integers to apply set partitioning property for proposed primitive Lipschitz integers. For $\mathcal{L}_{5+3 i+2 j+k}$ proposed Lipschitz constellation, the minimum squared Euclidean distance, CFM and average energy are 1, 12.9744 and 0.1542, respectively. The $\mathcal{L}_{5+3 i+2 j+k}$ proposed Lipschitz constellation is partition the $c=3$ different subsets with each set $d=13$ elements. So, the SNR coding gain of $\mathcal{L}_{5+3 i+2 j+k}$ proposed Lipschitz constellation is computed by using a subset of $\mathcal{L}_{5+3 i+2 j+k}$ proposed Lipschitz constellation. This subset is the set $\mathcal{L}_{5+3 i+2 j+k}^{(0)}$ with 13 elements. Because, the minimum squared Euclidean distance of $\mathcal{L}_{5+3 i+2 j+k}^{(0)}$ constellation is larger than the minimum squared Euclidean distance of $\mathcal{L}_{5+3 i+2 j+k}^{(1)}$ and $\mathcal{L}_{5+3 i+2 j+k}^{(2)}$ constellations. The minimum squared Euclidean distance, CFM and average energy of $\mathcal{L}_{5+3 i+2 j+k}^{(0)}$ Lipschitz signal constellation that is a subset of $\mathcal{L}_{5+3 i+2 j+k}$ Lipschitz constellation are 9, 12.9231 and 1.3929, respectively. Also, the proposed primitive Lipschitz integer used to construct the Lipschitz constellation that is the equivalence $\mathcal{L}_{5+3 i+2 j+k}^{(0)}$ constellation with 13 elements is $2+2 i+2 j+k$ (see Table III). The minimum squared Euclidean distance, CFM and average energy of $\mathcal{L}_{2+2 i+2 j+k}$ Lipschitz constellation is $1,0.4643$, and 4.3077 . The minimum squared Euclidean distance, CFM and average energy of the Gaussian constellation $\mathcal{G}_{3+2 i}$ with 13 elements are $1,2.1539$ and 0.4643 , respectively. Therefore, SNR gain of $\mathcal{L}_{5+3 i+2 j+k}$ proposed Lipschitz constellation is

$$
\mathrm{SNR}=-10 \log \left(\frac{\mathrm{CFM} \text { of } \mathcal{L}_{5+3 i+2 j+k}^{(0)}}{\mathrm{CFM} \text { of } \mathcal{L}_{2+2 i+2 j+k}}\right)=-10 \log \left(\frac{1.3929}{0.4643}\right)=4.77 .
$$

For $\mathcal{L}_{6+i+j+k}$ proposed Lipschitz constellation, the minimum squared Euclidean distance, CFM and average energy are 1, 12.9744 and 0.1542 , respectively. The $\mathcal{L}_{6+i+j+k}$ proposed Lipschitz constellation is partition the $c=3$ different subsets with each set $d=13$ elements. So, the SNR coding gain of $\mathcal{L}_{6+i+j+k}$ proposed Lipschitz constellation is computed by using a subset of $\mathcal{L}_{6+i+j+k}$ proposed Lipschitz constellation. This subset is the set $\mathcal{L}_{6+i+j+k}^{(0)}$ with 13 elements. Because, the minimum squared Euclidean distance of $\mathcal{L}_{6+i+j+k}^{(0)}$ is larger than the minimum squared Euclidean distance of $\mathcal{L}_{6+i+j+k}^{(1)}$ and $\mathcal{L}_{6+i+j+k}^{(2)}$. The minimum squared Euclidean distance, CFM and average energy of $\mathcal{L}_{6+i+j+k}^{(0)}$ Lipschitz signal constellation that is a subset of $\mathcal{L}_{6+i+j+k}$ proposed Lipschitz constellation are 3, 12.9231 and 0.4643, respectively. The average energy, CFM and minimum squared Euclidean distance of $\mathcal{L}_{5+3 i+2 j+k}$ and $\mathcal{L}_{6+i+j+k}$ proposed Lipschitz constellations have the same. But the subsets of these proposed Lipschitz constellations are have same average energy but they have different the values of CFM and the minimum squared Euclidean distance. So, the proposed primitive Lipschitz integer used to construct proposed Lipschitz constellation that has higher 
CFM and larger minimum squared Euclidean distance between subsets of $\mathcal{L}_{5+3 i+2 j+k}$ and $\mathcal{L}_{6+i+j+k}$ proposed Lipschitz constellations is $5+3 i+2 j+k$. For transmission over AWGN channel, the $\mathcal{L}_{5+3 i+2 j+k}$ proposed Lipschitz signal constellation has better performance than the $\mathcal{L}_{6+i+j+k}$ proposed Lipschitz signal constellation.

Example 4.6. We consider proposed Lipschitz constellation(s) with $N=$ $6 \cdot 25=150$ elements. There exist eight proposed primitive Lipschitz integers used to construct proposed Lipschitz constellations with $N=150$. These primitive Lipschitz integers are $7+7 i+6 j+4 k, 8+6 i+5 j+5 k$, $8+7 i+6 j+k, 9+7 i+4 j+2 k, 9+8 i+2 j+k, 10+5 i+4 j+3 k$, $11+4 i+3 j+2 k$ and $12+2 i+j+k$. There is no Gaussian constellation that has an equal size with the proposed Lipschitz constellation that has $N=150$ elements. We consider $10+5 i+4 j+3 k$ and $11+4 i+3 j+2 k$ proposed primitive Lipschitz integers between these proposed primitive Lipschitz integers. For $\mathcal{L}_{10+5 i+4 j+3 k}$ proposed Lipschitz constellation, the minimum squared Euclidean distance, CFM and average energy are 1, 49.98 and 0.4000, respectively. The $\mathcal{L}_{10+5 i+4 j+3 k}$ proposed Lipschitz constellation is partition the $c=6$ different subsets with each set $d=25$ elements. So, the SNR coding gain of $\mathcal{L}_{10+5 i+4 j+3 k}$ proposed Lipschitz constellation is computed by using a subset of $\mathcal{L}_{10+5 i+4 j+3 k}$ proposed Lipschitz constellation. This subset is the set $\mathcal{L}_{10+5 i+4 j+3 k}^{(0)}$ with 25 elements. Because, the minimum squared Euclidean distance of $\mathcal{L}_{10+5 i+4 j+3 k}^{(0)}$ constellation is larger than the minimum squared Euclidean distance of $\mathcal{L}_{10+5 i+4 j+3 k}^{(1)}, \mathcal{L}_{10+5 i+4 j+3 k}^{(2)}, \mathcal{L}_{10+5 i+4 j+3 k}^{(3)}, \mathcal{L}_{10+5 i+4 j+3 k}^{(4)}$, and $\mathcal{L}_{10+5 i+4 j+3 k}^{(5)}$ constellations. The minimum squared Euclidean distance, CFM and average energy of $\mathcal{L}_{10+5 i+4 j+3 k}^{(0)}$ Lipschitz signal constellation that is a subset of $\mathcal{L}_{10+5 i+4 j+3 k}$ proposed Lipschitz constellation are 30, 48.96 and 1.2255, respectively. Also, the Lipschitz integer used to construct the Lipschitz constellation that is the equivalence $\mathcal{L}_{10+5 i+4 j+3 k}^{(0)}$ constellation with 25 elements is $4+2 i+2 j+k$ (see Table III). The minimum squared Euclidean distance, CFM and average energy of $\mathcal{L}_{4+2 i+2 j+k}$ constellation is $1,0.2404$, and 8.3200. The minimum squared Euclidean distance, CFM and average energy of the Gaussian constellation $\mathcal{G}_{3+2 i}$ with 13 elements are 1, 2.1539 and 0.4643 , respectively. Therefore, SNR gain of $\mathcal{L}_{10+5 i+4 j+3 k}$ proposed Lipschitz constellation is

$$
\mathrm{SNR}=-10 \log \left(\frac{\mathrm{CFM} \text { of } \mathcal{L}_{10+5 i+4 j+3 k}^{(0)}}{\mathrm{CFM} \text { of } \mathcal{L}_{4+2 i+2 j+k}}\right)=-10 \log \left(\frac{1.2255}{0.2404}\right)=7.07 .
$$

For $\mathcal{L}_{11+4 i+3 j+2 k}$ proposed Lipschitz constellation, the minimum squared Euclidean distance, CFM and average energy of this primitive Lipschitz integer are 1, 50.0067 and 0.0400 , respectively. The $\mathcal{L}_{11+4 i+3 j+2 k}$ proposed Lipschitz constellation is partition the $c=6$ different subsets with each set $d=25$ elements. So, the SNR coding gain of $\mathcal{L}_{11+4 i+3 j+2 k}$ proposed Lipschitz 
constellation is computed by using a subset of $\mathcal{L}_{11+4 i+3 j+2 k}$ proposed Lipschitz constellation. This subset is the set $\mathcal{L}_{11+4 i+3 j+2 k}^{(0)}$ with 25 elements. Because, the minimum squared Euclidean distance of $\mathcal{L}_{11+4 i+3 j+2 k}^{(0)}$ is larger than the minimum squared Euclidean distance of $\mathcal{L}_{11+4 i+3 j+2 k}^{(1)}$ and $\mathcal{L}_{11+4 i+3 j+2 k}^{(1)}$, $\mathcal{L}_{11+4 i+3 j+2 k}^{(3)}, \mathcal{L}_{11+4 i+3 j+2 k}^{(4)}$ and $\mathcal{L}_{11+4 i+3 j+2 k}^{(5)}$. The minimum squared Euclidean distance, CFM and average energy of $\mathcal{L}_{11+4 i+3 j+2 k}^{0}$ Lipschitz signal constellation that is a subset of $\mathcal{L}_{11+4 i+3 j+2 k}$ proposed Lipschitz constellation are 30, 49.92 and 1.2019, respectively. The $\mathcal{L}_{10+5 i+4 j+3 k}$ and $\mathcal{L}_{11+4 i+3 j+2 k}$ proposed Lipschitz constellations have the same CFM and minimum squared Euclidean distance but average energies are not same. On the other hand, the subsets of these proposed Lipschitz constellations are have different average energies and CFM but the minimum squared Euclidean distances are the same. So, the proposed primitive Lipschitz integer used to construct the proposed Lipschitz constellation that has higher CFM and minimum average energy between subsets of $\mathcal{L}_{10+5 i+4 j+3 k}$ and $\mathcal{L}_{11+4 i+3 j+2 k}$ proposed Lipschitz constellations is $10+5 i+4 j+3 k$. for transmission over AWGN channel, the $\mathcal{L}_{10+5 i+4 j+3 k}$ proposed Lipschitz constellation has better performance than the $\mathcal{L}_{11+4 i+3 j+2 k}$ proposed Lipschitz constellation.

\section{Conclusion}

In this study, we investigated Lipschitz integers that have "division with small remainder" property and defined a new set, which is formed Lipschitz integers that have "division with small remainder" property, named encoder Lipschitz integers. So, we can define a Euclidean metric for Lipschitz constellations that lies on the Lipschitz integers or, different appropriate metrics for codes over the rings of Lipschitz integers. We showed that the Euclid division algorithm whether works or not for Lipschitz integers with proposition 3.1 and proposition 3.2. We can check whether the Euclidean division algorithm works for Lipschitz integers with these propositions (see example 4.1 and example 4.2). Also, we examined the performances of the Lipschitz integers, which have the "division with small remainder" property, for transmission over the AWGN channel, and proposed new Lipschitz constellations for transmission over AWGN channel (see Table I).

\section{References}

[1] Ian F. Blake, "Codes over certain rings," Information and Control, Volume 20, Issue 4, Pages 396-404, May 1972.

[2] Ian F. Blake, "Codes over integer residue rings," Information and Control, Volume 29, Issue 4, Pages 295-300, December 1975.

[3] Eugene Spiegel, "Codes over $\mathbb{Z}_{m}$," Information and Control, Volume 35, Issue 1, Pages 48-51, September 1977. 
[4] Eugene Spiegel, "Codes over $\mathbb{Z}_{m}$ (revisited)," Information and Control, Volume 37, Issue 1, Pages 100-104, April 1978.

[5] Chandra Satyanarayana, "Lee metric codes over integer residue rings (Corresp.)," IEEE Transactions on Information Theory, Volume 25, Issue 2, March 1979.

[6] A. R. Calderbank and N. J. A. Sloane, "Modular and p-adic cyclic codes," Designs, Codes and Cryptography, Volume 6, Issue 1, Pages 21-35, July 1995.

[7] Mehmet Ozen and Murat Guzeltepe, "Cyclic codes over some finite rings," Selcuk Journal of Applied Mathematics, Volume 11, No. 2, Pages 71-76, 2010.

[8] K. Abdelmoumen, H. Ben-azza and M. Najmeddine, About Euclidean codes in rings, British Journal of Mathematics and Computer Science, Volume 4, Issue 10, Pages 1356-1364, 16-31 May 2014.

[9] Klaus Huber, "Codes over Gaussian integers," IEEE Transactions on Information Theory, Volume 40, Issue 1, pp 207-216, January 1994.

[10] Stefka Bouyuklieva, "Applications of the Gaussian integers in coding theory," Proceedings of the 3rd International Colloquium on Differential Geometry and its Related Fields, September 3-7,2012.

[11] Jurgen Freudenberger, Farhad Ghaboussi and Sergo Shavgulidze, "New coding techniques for codes over Gaussian integers," IEEE Transactions on Communications, Volume 61, Issue 8, Pages 3114-3124, August 2013.

[12] Jurgen Freudenberger, Farhad Ghaboussi and Sergo Shavgulidze, "Set Partitioning and Multilevel Coding for Codes Over Gaussian Integer Rings," SCC 2013; 9th International ITG Conference on Systems, Communication and Coding, Munchen, Deutschland, pp. 1-5, 21-24 January 2013.

[13] Mehmet Ozen and Murat Guzeltepe, "Codes over Quaternion Integers," European Journal of Pure and Applied Mathematics, Volume 3, No. 4, Pages 670-677, July 2010

[14] J. Freudenberger and S. Shavgulidze, "New Signal Constellations for Coding Over Lipschitz Integers," SCC 2015; 10th International ITG Conference on Systems, Communications and Coding, Hamburg, Germany, Pages 1-6, 2-5 February 2015

[15] Jurgen Freudenberger and Sergo Shavgulidze, "New four-dimensional signal constellations from Lipschitz integers for transmission over the Gaussian channel," IEEE Transactions on Communications, Volume 63, Issue 7, Page 24202427, July 2015.

[16] Mehmet Ozen and Murat Guzeltepe, "Cyclic codes over some finite quaternion integer rings," Journal of the Franklin Institute, Volume 348, Issue 7, Pages 1312-1317, September 2011.

[17] Carmen Martinez, Ramon Beivide and Ernst M. Gabidulin, "Perfect codes from Cayley graphs over Lipschitz integers," IEEE Transactions on Information Theory, Volume 55, Issue 8, Pages 3552-3562, August 2009.

[18] Tariq Shah and Summera Said Rasool, "On codes over quaternion integers," Applicable Algebra in Engineering, Communication and Computing, Volume 24, Issue 6, December 2013.

[19] Young Ju Choie and Steven T. Dougherty, "Codes over $\mathbb{Z}_{2 m}$ and Jacobi forms over the quaternions," Applicable Algebra in Engineering, Communication and Computing, Volume 15, Issue 2, Pages 129-147, September 2004. 
[20] Murat Guzeltepe and Olof Heden, "Perfect Mannheim, Lipschitz and Hurwitz weight codes," Mathematical Communications, Volume 19, No. 2, Pages 253276, 2014.

[21] Giuliana Davidoff, Peter Sarnak and Alain Valette, "Elementary Number Theory, Group Theory, and Ramanujan Graphs," Cambridge University Press, 2003.

[22] John Horton Conway and Derek Alan Smith, "On Quaternions and Octonions: Their Geometry, Arithmetic, and Symmetry," Natick, MA, USA: A.K. Peters Ltd., 2003.

[23] G. D. Forney and L.-F. Wei, "Multidimensional constellations. I. Introduction, figures of merit, and generalized cross constellations," IEEE IEEE Journal on Selected Areas in Communications, Volume 7, No. 6, Pages 877-892, August 1989.

[24] J. Freudenberger and S. Shavgulidze, "New signal constellations for coding over Lipschitz integers," in Proceedings of 10th International ITG Conference on Systems, Communications and Coding (SCC 2015), Pages 1-6, February 2015 .

\author{
Ramazan Duran \\ Department Mathematics, \\ Faculty of Arts and Sciences, \\ TR 03200 Afyonkarahisar, \\ Turkey \\ e-mail: rduran@aku.edu.tr \\ Department Mathematics, \\ Faculty of Arts and Sciences, \\ TR 54187 Sakarya, \\ Turkey \\ e-mail: ramazan.duran1@ogr.sakarya.edu.tr \\ Murat Guzeltepe \\ Department Mathematics, \\ Faculty of Arts and Sciences, \\ TR 54187 Sakarya, \\ Turkey \\ e-mail: mguzeltepe@sakarya.edu.tr
}

